\title{
Generic diffeomorphisms on compact surfaces
}

\author{
by \\ Flavio Abdenur (Rio de Janeiro), Christian Bonatti (Dijon), \\ Sylvain Crovisier (Paris) and Lorenzo J. Díaz (Rio de Janeiro)
}

\begin{abstract}
We discuss the remaining obstacles to prove Smale's conjecture about the $C^{1}$-density of hyperbolicity among surface diffeomorphisms. Using a $C^{1}$-generic approach, we classify the possible pathologies that may obstruct the $C^{1}$-density of hyperbolicity. We show that there are essentially two types of obstruction: (i) persistence of infinitely many hyperbolic homoclinic classes and (ii) existence of a single homoclinic class which robustly exhibits homoclinic tangencies. In the course of our discussion, we obtain some related results about $C^{1}$-generic properties of surface diffeomorphisms involving homoclinic classes, chain-recurrence classes, and hyperbolicity. In particular, it is shown that on a connected surface the $C^{1}$-generic diffeomorphisms whose non-wandering sets have non-empty interior are the Anosov diffeomorphisms.
\end{abstract}

\section{INTRODUCTION}

1.1. Motivations. Some ideas of Mañé and new $C^{1}$-perturbation lemmas generalizing Hayashi's connecting lemma $[\mathrm{H}]$ have recently opened the door for a global understanding of $C^{1}$-generic systems (diffeomorphisms or flows) on compact manifolds of any dimension. Many mechanisms responsible for robust non-hyperbolicity are now understood. It is not known, however, whether the best-known $C^{2}$-mechanism for generating robust nonhyperbolic behavior, the Newhouse phenomenon (of persistence of tangencies and coexistence of infinitely many sinks), has some $C^{1}$-equivalent in dimension two. In fact, this seems the most important open problem for the global understanding of the $C^{1}$-generic systems on compact surfaces: it remains unknown whether the (open) set of Axiom A diffeomorphisms is

2000 Mathematics Subject Classification: 37C05, 37C20, 37C25, 37C29, 37C70.

Key words and phrases: chain-recurrence class, dominated splitting, filtration, homoclinic class, hyperbolicity, surface diffeomorphism.

This work was partially supported by the Agreement in Mathematics Brazil - France, CAPES/Prodoc, CNPq, Faperj, and Pronex (Brazil). The authors acknowledge the kind hospitality of IMB - Univ. de Bourgogne (France) and IMPA (Brazil) during their visits while preparing this paper. 
dense in the space of surface diffeomorphisms, for the $C^{1}$-topology. We do expect a positive answer to the following conjecture:

Conjecture 1 (Smale, $[\mathrm{Sm}]$ ). Let $S$ be a compact surface. The set of diffeomorphisms satisfying Axiom $A$ and the no-cycle condition is (open and) dense in $\operatorname{Diff}^{1}(S)$.

This conjecture is known to be false in dimension 3 or higher, for the $C^{1}$-topology, due to the co-existence of hyperbolic saddles with different indices (dimension of the unstable bundle) in the same transitive set (see [AS] and [Si] for counter-examples in dimension 4 and 3) and in dimension 2 or higher for the $C^{r}$-topology, $r \geq 2$, due to the phenomenon of persistence of tangencies (see $[\mathrm{N}]$ and $[\mathrm{PV}]$ ).

Here we try to present an overview of the dynamics of $C^{1}$-generic diffeomorphisms of compact surfaces far from hyperbolic systems. If one believes in Smale's conjecture above, one can say that this work discusses the remaining obstacles to proving the conjecture. The difficulty of the conjecture comes from the fact that we do not know any local mechanism which could be responsible for robust non-hyperbolicity in dimension 2 for the $C^{1}$-topology (in fact, we expect that such a mechanism does not exist). For diffeomorphisms in higher dimensions, the only local phenomenon which generates $C^{1}$-robust non-hyperbolicity is associated to the unfolding of heterodimensional cycles (i.e., cycles associated to saddles having different indices); see $[D, D R]$. Of course, such a cycle may not appear on surfaces and the main candidate for such a mechanism on surfaces seems to be the presence of homoclinic tangencies, i.e. non-transverse intersections between the invariant manifolds of some hyperbolic periodic orbit: for the $C^{2}$-topology, they do imply the existence of a $C^{2}$-open set of non-hyperbolic diffeomorphisms. Moreover, Pujals and Sambarino proved in [PS] that the existence of homoclinic tangencies is a $C^{1}$-dense phenomenon among surface diffeomorphisms far from hyperbolicity (i.e. diffeomorphisms that cannot be approximated by hyperbolic ones).

Since the result of [PS] gives only a description for a dense part of the set of diffeomorphisms far from hyperbolicity and since we are interested in robust non-hyperbolic phenomena, our aim in this paper is to study a generic part of this set of diffeomorphims (meaning a residual subset of $\left.\operatorname{Diff}^{1}(M)\right)$. By $[\mathrm{PS}]$, we know that surface diffeomorphisms that are far from hyperbolicity may be perturbed to create homoclinic tangencies. However, it is not clear whether such tangencies can be obtained in a persistent way for the same periodic point. This motivates the following definition:

Definition 1.1. A diffeomorphism $f$ of a compact manifold $M$ has a persistent homoclinic tangency associated to a hyperbolic periodic point $p$ if there exist a $C^{1}$-neighborhood $\mathcal{U}$ of $f$ and a dense subset $\mathcal{D} \subset \mathcal{U}$ such that 
for any diffeomorphism $g \in \mathcal{D}$ the continuation $p_{g}$ of $p$ exhibits a homoclinic tangency.

The following consequence of our results explains what occurs for generic diffeomorphisms (i.e. diffeomorphisms in a residual subset) far from hyperbolicity. Such a diffeomorphism may (in principle) exhibit a persistent tangency, but we cannot exclude a priori another possibility: that the homoclinic class of any periodic orbit is a hyperbolic basic set but that the global dynamics exhibits infinitely many different homoclinic classes accumulating on aperiodic classes (see Section 1.2 below for the definition of aperiodic class).

TheOREM 1. Let $S$ be a closed surface. There are three disjoint open sets $\mathcal{H}, \mathcal{T}$, and $\mathcal{W}$, whose union is dense in $\operatorname{Diff}^{1}(S)$, such that:

- $\mathcal{H}$ is the set of diffeomorphisms which satisfy Axiom A and the no-cycle condition;

- $\mathcal{T}$ is the set of diffeomorphisms admitting a persistent homoclinic tangency associated to some hyperbolic periodic saddle;

- $\mathcal{W}$ contains a residual subset $\mathcal{G W}$ such that every $f \in \mathcal{G W}$ has infinitely many homoclinic classes, all of which are hyperbolic basic sets.

Let us now discuss how homoclinic tangencies associated to a single homoclinic class may occur persistently. Keeping in mind the classical $C^{2}$ Newhouse phenomenon (see $[\mathrm{N}]$ ), the most intuitive way is to imagine that some hyperbolic set has a robust tangency in the following sense:

Definition 1.2. Let $\Lambda$ be a hyperbolic set of a diffeomorphism $f$ on a compact manifold. We say that $\Lambda$ has robust tangencies if there is a constant $T>0$ and a $C^{1}$-neighborhood $\mathcal{U}$ of $f$ such that for any $g \in \mathcal{U}$ the local stable manifold $W_{T}^{\mathrm{s}}\left(\Lambda_{g}\right)$ of size $T$ of the continuation $\Lambda_{g}$ of $\Lambda$ is tangent to the local unstable manifold $W_{T}^{\mathrm{u}}\left(\Lambda_{g}\right)$ of size $T$ of $\Lambda_{g}$.

In fact, if a hyperbolic set $\Lambda$ has locally generically some tangency then it has robust tangencies:

Proposition 1.3. Let $f$ be a diffeomorphism of a compact manifold $M$ and $\Lambda$ a hyperbolic set of $f$. If there exist a neighborhood $\mathcal{U}$ of $f$ in $\operatorname{Diff}^{1}(M)$ and a residual subset $\mathcal{G}$ of $\mathcal{U}$ such that for every diffeomorphism $g \in \mathcal{G}$ the stable and unstable manifolds of the continuation $\Lambda_{g}$ of $\Lambda$ are tangent, then $f$ is $C^{1}$-approached by diffeomorphisms $h$ which exhibit robust tangencies.

It is natural to ask whether a diffeomorphism in $\mathcal{T}$ (i.e., one that has a persistent homoclinic tangency) admits robust tangencies. The following proposition explains what else might happen.

Proposition 1.4. There are two $C^{1}$-open disjoint subsets $\mathcal{T}_{\text {rob }}$ and $\mathcal{T}_{\infty}$ of $\mathcal{T}$, whose union is dense in $\mathcal{T}$, such that: 
- $\mathcal{T}_{\text {rob }}$ is the set of diffeomorphisms admitting a robust tangency associated to some hyperbolic set;

- there is a residual subset $\mathcal{G} \mathcal{T}_{\infty}$ in $\mathcal{T}_{\infty}$ such that for every $g \in \mathcal{G} \mathcal{T}_{\infty}$ and for any hyperbolic set $\Lambda$ of $g$, the invariant manifolds of $\Lambda$ are transverse (even though $g$ has persistent tangencies in the sense of Definition 1.1).

When trying to solve Smale's Conjecture 1 above, one usually focuses on whether the Newhouse phenomenon (robust tangencies) can occur. The discussion above shows that two other phenomena may be responsible for the non-density of Axiom A diffeomorphisms: the creation of homoclinic tangencies either "at the infinity of some homoclinic class" (corresponding to the set $\mathcal{T}_{\infty}$ ) or "in the aperiodic classes" (corresponding to the set $\mathcal{W}$ ). We have therefore broken Smale's conjecture into three parts:

CONJECTURE 1. A generic diffeomorphism $\left({ }^{1}\right)$ of a compact surface whose homoclinic classes are all hyperbolic satisfies Axiom A.

ConjeCture 2 (No robust tangencies). Let $\Lambda$ be a hyperbolic set of a diffeomorphism $f$ of a compact surface $S$. Then, for any $L>0$, there is a $C^{1}$-perturbation $g$ of $f$ such that the local invariant manifolds of size $L$ of the hyperbolic continuation $\Lambda_{g}$ of $\Lambda$ for $g$ are transverse.

Conjecture 3 (Persistent tangencies imply robust tangencies). Any diffeomorphism $f$ which admits persistent tangencies associated to some hyperbolic periodic point may be $C^{1}$-approximated by a diffeomorphism which has robust tangencies associated to some hyperbolic set.

By Theorem 1, these three conjectures imply Smale's conjecture: Conjecture 1 implies that $\mathcal{W}$ is empty, Conjecture 2 implies that $\mathcal{T}_{\text {rob }}$ is empty, and finally, Conjecture 3 shows that the open set $\mathcal{T}_{\text {rob }}$ is dense in $\mathcal{T}$, implying that $\mathcal{T}_{\infty}$ is empty.

The third conjecture is equivalent to the following (a priori) weaker statement (see Proposition 6.2 at the end of the article):

CONJECTURE 4. Let $f$ be a $C^{1}$-generic diffeomorphism such that for any hyperbolic set $\Lambda$ of $f$ the stable and unstable manifolds of $\Lambda$ are transverse. Then all the homoclinic classes of $f$ are hyperbolic.

We end this discussion with another question related to the techniques of the paper. In the context of $C^{1}$-generic dynamics of diffeomorphisms in any dimension, $[\mathrm{ABD}]$ formulates, and proves in some particular cases, the following conjecture:

$\left({ }^{1}\right)$ Henceforward, "A generic diffeomorphism $f$ satisfies..." shall mean "There exists a residual subset $\mathcal{R}$ of $\operatorname{Diff}^{1}(S)$ such that every $f \in \mathcal{R}$ satisfies..." 
CONJECtuRE 5. For any $C^{1}$-generic diffeomorphism of a compact connected manifold, if the non-wandering set has non-empty interior then the diffeomorphism is transitive, in particular, the non-wandering set coincides with the whole manifold.

Notice that a positive answer to Smale's Conjecture 1 would imply a positive answer to this one in dimension two. The results proven in this paper do imply:

COROLlary 1. Let $S$ be a connected closed surface. There is a residual subset $\mathcal{G}_{0}$ of $\operatorname{Diff}^{1}(S)$ consisting of diffeomorphisms $f$ such that

$$
\operatorname{int}(\Omega(f)) \neq \emptyset \Rightarrow S \text { is the torus } T^{2} \text { and } f \text { is Anosov. }
$$

Moreover, in this case $f$ is topologically conjugate to some linear Anosov diffeomorphism. In particular, $f$ is transitive.

The conjugacy to a linear Anosov system in the previous corollary is a consequence of a result of Franks [F]. Thus in this paper we settle the two-dimensional case of the conjecture in $[\mathrm{ABD}]$.

We note that the residual set $\mathcal{G}_{0}$ of Corollary 1 will be given by Theorem 2 below.

1.2. Presentation of the results. For surface diffeomorphisms, Pujals and Sambarino have provided in [PS] a precise description of the dynamics under the hypothesis of the existence of a dominated splitting. This notion will be important for us since from a $C^{1}$-generic viewpoint it characterizes hyperbolicity.

Definition 1.5. A compact set $K$ which is invariant under a diffeomorphism $f$ admits a dominated splitting if the tangent bundle $T_{K} M$ over $K$ splits into two bundles, $T_{K} M=E \oplus F$, and there exists an integer $l \geq 1$ such that for every point $x \in K$ and every pair of unit vectors $u \in E$ and $v \in F$ we have

$$
\left\|D f^{l}(x) \cdot u\right\| \leq 2\left\|D f^{l}(x) \cdot v\right\| .
$$

The next remark states the main properties of a dominated splitting we use in this paper (see, for instance, [BDV, Section B.1.1]).

REMARK 1.6 (Properties of dominated splittings). Let $\Lambda$ be an $f$-invariant set with a dominated splitting $E \oplus F$.

(1) Extension to the closure. The dominated splitting $E \oplus F$ can be extended in a dominated way to the closure of $\Lambda$.

(2) Extension to a neighborhood. The dominated splitting $E \oplus F$ can be extended in a dominated way to the maximal invariant set of $f$ in a small neighborhood of $\Lambda$. 
(3) Robustness. Every dominated splitting persists under $C^{1}$-perturbations: There are a $C^{1}$-neighborhood $\mathcal{U}$ of $f$ and a neighborhood $U$ of $\Lambda$ in the ambient manifold such that, for every $g \in \mathcal{U}$, the maximal invariant set of $g$ in $U$ has a dominated splitting.

Another important ingredient for what follows is the decomposition of the dynamics into its chain-recurrence classes, given by Conley's theory [Co]. We recall the main results of this theory and state some relevant properties of chain-recurrence classes in Section 2.1.

Let $f$ be a homeomorphism of a compact metric space $X$. For any $\varepsilon>0$, an $\varepsilon$-pseudo-orbit of $f$ is a sequence $\left(x_{n}\right)$ in $X$ such that, for each $n$, $d\left(f\left(x_{n}\right), x_{n+1}\right)<\varepsilon$. A point $x$ is chain-recurrent if for any $\varepsilon>0$ there exists an $\varepsilon$-pseudo-orbit starting and ending at $x$. We denote by $\mathcal{R}(f)$ the set of chain-recurrent points. It decomposes as the union of chain-recurrence classes, which are pairwise invariant compact sets defined as follows: two points $x$ and $y$ belong to the same chain-recurrence class if for any $\varepsilon>0$, there are $\varepsilon$-pseudo-orbits starting at $x$ and ending at $y$, and conversely starting at $y$ and ending at $x$.

A third important ingredient in this paper is the notion of homoclinic class. The homoclinic class of a hyperbolic periodic orbit $p$ of a diffeomorphism $f$, denoted by $H(p, f)$, is the closure of the transverse intersections of its stable and unstable manifolds. This set coincides with the closure of the periodic orbits $q$ homoclinically related to $p$, i.e., the stable manifold of $p$ transversely meets the unstable one of $q$, and the unstable manifold of $p$ transversely meets the stable one of $q$. We observe that a homoclinic class is always an $f$-invariant transitive set. An important property of homoclinic classes of $C^{1}$-generic diffeomorphisms is the following: any two homoclinic classes either coincide or are disjoint (see $[\mathrm{CMP}]$ ).

Quite recently, $[\mathrm{BC}]$ proved a perturbation lemma for pseudo-orbits and deduced from this lemma that for $C^{1}$-generic diffeomorphisms of compact manifolds, any chain-recurrence class which contains a hyperbolic periodic point $p$ coincides with the homoclinic class of $p$ (in particular, this result generalizes the one in $[\mathrm{CMP}]$ above). The other chain-recurrence classes of these generic diffeomorphisms (when they exist) are called aperiodic classes (whose existence in some locally generic regions was proven in $\left[\mathrm{BD}_{2}\right]$ ).

Note that for diffeomorphisms $f$ which satisfy Axiom $A$ (the non-wandering set $\Omega(f)$ is hyperbolic and equal to the closure of the periodic points) Smale has proven in his spectral theorem $[\mathrm{Sm}]$ that the non-wandering set is the union of finitely many pairwise disjoint homoclinic classes. These homoclinic classes are the basic sets of the spectral decomposition of the non-wandering set. Moreover, under the no-cycles assumption (i.e., there are no basic sets of the spectral decomposition cyclically related by inter- 
sections of the corresponding stable and unstable manifolds), the chainrecurrence classes are homoclinic classes. More precisely, in this case, each chain-recurrence class $E$ is a basic set:

- $E$ is a hyperbolic homoclinic class;

- $E$ is the maximal invariant set in a level of a filtration: there exist compact sets $U$ and $V$ such that $f(U) \subset \operatorname{int}(U), f^{-1}(V) \subset \operatorname{int}(V)$ and $E$ is the maximal invariant set in $U \cap V$, i.e. $E=\bigcap_{n \in \mathbb{Z}} f^{n}(U \cap V)$.

Our next result shows that, $C^{1}$-generically, a chain-recurrence class of a surface diffeomorphism is hyperbolic if, and only if, it is isolated, and also if, and only if, it admits a dominated splitting (this assertion does not hold in higher dimensions, see the series of examples in $\left.\left[\mathrm{Sh}, \mathrm{M}_{1}, \mathrm{BD}_{1}, \mathrm{BV}\right]\right)$ :

TheOREM 2. Let $S$ be a compact surface. There is a residual subset $\mathcal{G}_{0}$ of $\operatorname{Diff}^{1}(S)$ such that, for any $f \in \mathcal{G}_{0}$ and any chain-recurrence class $E$ of $f$, we have the following dichotomy: either

(1) $E$ is isolated in the chain-recurrent set $\mathcal{R}(f)$ of $f$; in this case, $E$ is a hyperbolic homoclinic class (and so a basic set); or

(2) $E$ is not isolated; in this case:

(a) E does not admit any dominated splitting;

(b) $E$ is contained in the closure of the set of sinks and sources of $f$;

(c) for any neighborhood $U$ of $E$, there is a $C^{1}$-neighborhood $\mathcal{U}$ of $f$ and a dense subset $\mathcal{D}$ of $\mathcal{U}$ such that any $g \in \mathcal{D}$ has a hyperbolic periodic point $p_{g}$ whose homoclinic class is contained in $U$ and has homoclinic tangencies.

If the chain-recurrence class $E$ is a non-isolated homoclinic class $H(p, f)$, we obtain the announced tangencies inside the homoclinic class $H\left(p_{g}, g\right)$ of the continuation $p_{g}$ of $p$ :

THEOREM 3. Let $f$ be a diffeomorphism of a compact surface $S$ and $p$ be a hyperbolic periodic saddle of $f$. If the homoclinic class $H(p, f)$ of $p$ has no dominated splitting, then there exists a diffeomorphism $g$ arbitrarily $C^{1}$-close to $f$ such that the continuation $p_{g}$ of $p$ admits a homoclinic tangency.

From the previous two theorems one deduces the following genericity result:

Corollary 2. Let $S$ be a compact surface. There is a residual subset $\mathcal{G}_{1}$ of $\operatorname{Diff}^{1}(S)$ such that, for every $f \in \mathcal{G}_{1}$ and every periodic point $p$ of $f$ whose homoclinic class is non-isolated in the recurrent set $\mathcal{R}(f)$ of $f$, the diffeomorphism $f$ exhibits a persistent homoclinic tangency associated to $p$. 


\section{SOME RESULTS IN ARBITRARY DIMENSION}

This section collects some results that we will use later in this paper. Most of them were obtained in previous works. In Section 2.1, we recall Conley's Fundamental Theorem of Dynamical Systems and state properties of chain-recurrence classes and their filtrations. A general reference for Conley's theory is $[R]$. In Section 2.2, we briefly study hyperbolic chain-recurrence classes. Finally, in Section 2.3, we state a result about generic chain-recurrent sets (which follows from a perturbation lemma for pseudo-orbits in [BC]) and deduce properties of non-isolated chain-recurrence classes from it.

2.1. Conley's theory and filtrating sets. We begin with Conley's fundamental theorem:

Theorem 2.1 (Conley's Fundamental Theorem of Dynamical Systems, $[\mathrm{Co}])$. Let $f$ be a homeomorphism of a compact metric space $X$. There exists a continuous function $\varphi: X \rightarrow \mathbb{R}$ such that:

(1) $\varphi$ is a Lyapunov function: for any $x \in X, \varphi(f(x)) \leq \varphi(x)$;

(2) for any $x \in X, \varphi(f(x))=\varphi(x)$ is equivalent to $x \in \mathcal{R}(f)$;

(3) for any $x, y \in \mathcal{R}(f), \varphi(x)=\varphi(y)$ is equivalent to $x$ and $y$ belonging to the same chain-recurrence class;

(4) $\varphi(\mathcal{R}(f))$ is a totally disconnected compact subset of $\mathbb{R}$.

Such a function $\varphi$ will be called a Lyapunov function adapted to $\mathcal{R}(f)$.

REMARK 2.1. If $X$ is a smooth compact manifold, the function $\varphi$ may be assumed to be smooth.

A compact set $A$ is a trapping region for $f$ if $f(A)$ is contained in the interior of $A$. The maximal invariant set $\bigcap_{n \in \mathbb{N}} f^{n}(A)$ of $f$ in $A$ is the attractor associated to $A$. In the same way, $\bigcap_{n \in \mathbb{N}} f^{-n}(X \backslash A)=\bigcap_{n \in \mathbb{N}} \overline{f^{-n}(X \backslash A)}$ is the repellor associated to $A$. If $B$ is a trapping region for $f^{-1}$, we define its attractor and repellor as the attractor and repellor of the trapping region $\overline{X \backslash B}$ for $f$.

We say that a compact set $U$ is a filtrating set if there are two compact sets $A, B$ such that:

- $U=A \cap B$

- $f(A) \subset \operatorname{int}(A)$ (that is, $A$ is a trapping region for $f$ );

- $f^{-1}(B) \subset \operatorname{int}(B)$ (that is, $B$ is a trapping region for $f^{-1}$ ).

Let $K$ be some invariant compact set of $f$. A filtrating neighborhood of $K$ is a neighborhood of $K$ which is a filtrating set. An isolating filtrating neighborhood $U$ of $K$ is a filtrating neighborhood of $K$ whose maximal invariant set $\bigcap_{n \in \mathbb{Z}} f^{n}(U)$ is $K$. 
REMARK 2.2 .

(1) Let $U=A \cap B$ be a filtrating set (where $A$ and $B$ are trapping regions for $f$ and $f^{-1}$ respectively). Then the maximal invariant set of $f$ in $U$ is the intersection of the attractor of $A$ with the repellor of $B$.

(2) If $U$ is a filtrating set for $f$, then $U$ is also a filtrating set for any homeomorphism $g$ of $X$ which is $C^{0}$-close to $f$.

(3) If $\varphi$ is a Lyapunov function adapted to $\mathcal{R}(f)$, then for any $a, b \in$ $\mathbb{R} \backslash \varphi(\mathcal{R}(f))$ with $a<b$, the set $\varphi^{-1}([a, b])$ is a filtrating set.

(4) Let $U=A \cap B$ be a filtrating set defined as above. Then any compact set $V$ contained in the interior of $U$ and containing $f(U) \cap f^{-1}(U)$ is also a filtrating set. Moreover, the maximal invariant sets of $f$ in $U$ and in $V$ coincide. Indeed, $V$ may be written as an intersection

$$
V=A^{\prime} \cap B^{\prime}=(V \cup f(A)) \cap\left(V \cup f^{-1}(B)\right),
$$

where $A^{\prime}$ and $B^{\prime}$ are trapping regions for $f$ and $f^{-1}$, respectively.

(5) By the last item, if $X$ is a smooth compact manifold then $V$ can be chosen to be a smooth submanifold with boundary. Hence, we can replace any filtrating set by a filtrating set which is a smooth submanifold with boundary having the same maximal invariant set.

(6) By the definition of filtrating set, any chain-recurrence class which intersects a filtrating set is contained in the interior of the filtrating set.

(7) Let $K$ be the maximal invariant set in a filtrating set $U=A \cap B$ as above. Then, for any $i, j \in \mathbb{Z}, f^{i}(A) \cap f^{j}(B)$ is a filtrating set having $K$ as its maximal invariant set. Moreover, $\left\{f^{n}(A) \cap f^{-n}(B): n \in \mathbb{N}\right\}$ is a basis of neighborhoods of $K$, each of which is a filtrating set.

Proposition 2.3. Let $f$ be a homeomorphism of a compact metric space $X, E$ any chain-recurrent class of $f, \varphi$ a Lyapunov function adapted to $\mathcal{R}(f)$, and $U$ a neighborhood of $E$. Then, for every $\varepsilon>0$ small enough, the maximal invariant set in $V_{\varepsilon}=\varphi^{-1}([\varphi(E)-\varepsilon, \varphi(E)+\varepsilon])$ is contained in $U$.

Proof. Note that the set of maximal invariant sets $\Lambda_{\varepsilon}$ in $V_{\varepsilon}$ is a decreasing family as $\varepsilon$ goes to 0 . We just have to prove that the intersection $\Lambda=\bigcap_{\varepsilon} \Lambda_{\varepsilon}$ is contained in the interior of $U$ : this set is an invariant compact set on which $\varphi$ is equal to $\varphi(E)$. As a consequence, $\Lambda=E$.

Proposition 2.4. Let $f$ be a homeomorphism of a compact metric space $X$. Any chain-recurrence class $E$ of $f$ admits arbitrarily small filtrating neighborhoods.

Proof. Let $\varphi$ be a Lyapunov function adapted to $\mathcal{R}(f)$ and $U$ a neighborhood of $E$. By Proposition 2.3, for $\varepsilon$ small enough, the maximal invariant 
set $\Lambda_{\varepsilon}$ in $V_{\varepsilon}=\varphi^{-1}([\varphi(E)-\varepsilon, \varphi(E)+\varepsilon])$ is contained in $U$. We choose $\varepsilon$ such that $\varphi(E)-\varepsilon$ and $\varphi(E)+\varepsilon$ do not belong to $\varphi(\mathcal{R}(f)$ ) (this is possible since $\varphi(\mathcal{R}(f))$ has empty interior). By Remark $2.2(3), V_{\varepsilon}$ is a filtrating set. By Remark 2.2(7), we can choose a filtrating set which is an arbitrarily small neighborhood of $\Lambda_{\varepsilon}$, hence contained in $U$ and containing $E$.

Proposition 2.5. Let $f$ be a homeomorphism of a compact manifold $M$ such that its chain-recurrent set $\mathcal{R}(f)$ has non-empty interior. Then there exists a chain-recurrence class which has non-empty interior.

Proof. Consider a connected component $C$ of the interior of $\mathcal{R}(f)$ and a Lyapunov function $\varphi$ adapted to $\mathcal{R}(f)$. The image $\varphi(C)$ is connected and contained in $\varphi(\mathcal{R}(f))$, which is totally disconnected. So, $\varphi(C)$ is a point and, by Theorem 2.1, $C$ is contained in a chain-recurrence class $E$. Since a manifold is locally connected, $C$ has non-empty interior. Hence, $E$ has non-empty interior.

Proposition 2.6. Let $X$ be a compact metric space. There exists a countable family $\mathcal{F}=\left\{V_{n}\right\}$ of compact sets such that for any homeomorphism $f$ of $X$ and any filtrating set $U$ of $f$, there exists $V_{n} \subset U$ which is a filtrating set of $f$ such that the maximal invariant sets of $f$ in $U$ and in $V_{n}$ coincide.

Proof. Let $\mathcal{O}=\left\{O_{n}\right\}$ be a countable basis of open sets of $X$. We consider the countable family $\mathcal{F}$ of compact sets that are the closures of finite unions of sets in $\mathcal{O}$.

Let $f$ be a homeomorphism of $X$ and $U$ a filtrating set. We consider the compact set $f(U) \cap f^{-1}(U)$, contained in the interior of $U$. Each point $x$ of $f(U) \cap f^{-1}(U)$ belongs to some open set $O_{n(x)}$, whose closure is contained in the interior of $U$. Let $V$ be the closure of the union of a finite covering of $f(U) \cap f^{-1}(U)$ by such $O_{n(x)}$. The compact set $V$ belongs to $\mathcal{F}$, is contained in the interior of $U$, and contains $f(U) \cap f^{-1}(U)$. Thus, by Remark 2.2(4), the set $V$ is a filtrating set which has the same maximal invariant set as $U$.

Proposition 2.7. Let $f$ be a homeomorphism of a compact metric space $X$ and let $U$ be a filtrating set. Then there exist two trapping regions $A$ for $f$ and $B$ for $f^{-1}$ such that $U=A \cap B$ and the following three equivalent properties are satisfied:

(1) the attractor of $A$ contains the attractor of $B$;

(2) the repellor of $B$ contains the repellor of $A$;

(3) the repellor of $A$ is disjoint from the attractor of $B$.

Proof. We first prove that the three properties are equivalent. First, notice that the repellor associated to a trapping region is the largest invariant compact set disjoint from the attractor of the trapping region. If (1) is sat- 
isfied, the repellor of $A$ is disjoint from the attractor of $A$, hence of $B$ (i.e. (3) is satisfied). Conversely, if the repellor of $A$ is disjoint from the attractor of $B$ (item $(3)$ ), then it is contained in the largest invariant compact set disjoint from the attractor of $B$, that is, the repellor of $B$ (this gives (2)). The other implications follow similarly.

Let now $U$ be a filtrating set. By definition, there are two trapping regions $A_{0}$ for $f$ and $B$ for $f^{-1}$ such that $U=A_{0} \cap B$. We define $A$ as the union of $A_{0}$ with $f(X \backslash \operatorname{int}(B))$. Since the union of two trapping regions is a trapping region, $A$ and $B$ are trapping regions for $f$ and $f^{-1}$, respectively. Since $B$ is a trapping region for $f^{-1}, f(X \backslash \operatorname{int}(B))$ is disjoint from $B$ so that $A \cap B=A_{0} \cap B=U$.

Finally, the maximal invariant set in $A$ contains the maximal invariant set in $f(X \backslash \operatorname{int}(B))$. Hence, the attractor of $A$ contains the attractor of $B$.

2.2. Hyperbolic theory. The following result is certainly well known and has been proven, in particular, in [ABD, Theorem 1].

Proposition 2.8. Let $M$ be a connected compact manifold. Any hyperbolic set $\Lambda$ of a diffeomorphism $f \in \operatorname{Diff}^{1}(M)$, contained in the nonwandering set $\Omega(f)$ of $f$ and with non-empty interior, is the whole manifold. Thus the diffeomorphism $f$ is Anosov.

We recall that a transitive hyperbolic set which is the maximal invariant set in a neighborhood is called a basic set. A basic set which is a chainrecurrence class is called a basic piece.

From Theorem 2.1 and the hyperbolic theory, we get:

Proposition 2.9. Let $f$ be a diffeomorphism of a compact manifold. Any hyperbolic chain-recurrence class of $f$ is an isolated homoclinic class (hence, a basic piece of $f$ ).

REMARK 2.10. In fact, any chain-recurrence class containing a hyperbolic set contains a periodic point.

Proof. Let $E$ be a hyperbolic chain-recurrence class of $f$. From Proposition 2.4, $E$ admits arbitrarily small filtrating neighborhoods. Consider a small filtrating neighborhood $U$ of $E$. The maximal invariant set $\Lambda$ in $U$ is a compact hyperbolic set. Moreover, the shadowing lemma (see, for instance, $[\mathrm{Y}]$ ) asserts that, for any small $\delta$, any $\varepsilon$-pseudo-orbit, with $\varepsilon>0$ small enough, is $\delta$-shadowed by a unique orbit of $\Lambda$. One deduces that $\Lambda$ contains a sequence $\left\{\gamma_{n}\right\}$ of periodic orbits converging to $E$ in the Hausdorff topology. As $\Lambda$ is hyperbolic, the continuity of the local stable and unstable manifolds implies that there is $\mu>0$ such that any two periodic orbits in $\Lambda$ having points $\mu$-close to each other are homoclinically related, so they have the same homoclinic class. As a consequence, all the $\gamma_{n}$, for 
$n$ large enough, have the same homoclinic class $H$. As $H$ is compact (by definition) it contains $E$. However, $H$ is transitive so it is contained in a unique chain-recurrence class. Hence $E=H$, concluding the proof.

As a consequence of the previous proposition and the persistence of hyperbolic sets, one has the following:

Proposition 2.11. Let $f$ be a diffeomorphism of a compact manifold and $V$ a filtrating set. Suppose that $\mathcal{R}(f) \cap V$ is hyperbolic. Then:

- $\mathcal{R}(f) \cap V$ consists of finitely many basic pieces;

- there is a $C^{1}$-neighborhood of $f$ consisting of diffeomorphisms $g$ such that $V$ is a filtrating set of $g$ and $\mathcal{R}(g) \cap V$ consists of finitely many basic pieces which are the continuations of the basic pieces of $f$. In particular, $g$ on $\mathcal{R}(g) \cap V$ is topologically conjugate to $f$ on $\mathcal{R}(f) \cap V$.

2.3. Genericity results. $[\mathrm{BC}]$ states the following consequences of the connecting lemma for pseudo-orbits:

THEOREM 2.2. Let $M$ be a compact manifold. The set of diffeomorphisms $f$ for which all the periodic orbits are hyperbolic and the chainrecurrent set $\mathcal{R}(f)$ is the closure of the set of periodic points is a residual subset of $\operatorname{Diff}^{1}(M)$.

This is a direct consequence of Kupka-Smale's theorem, of Pugh's theorem on the generic density of the periodic points in the non-wandering set, $[\mathrm{P}]$, and of [BC, Corollaire 1.2].

Proposition 2.12 ([BC, Remarque 1.10, 1.12 and Corollaire 1.11]). There is a residual part $\mathcal{G}_{2}$ of $\operatorname{Diff}^{1}(M)$ such that for any $f \in \mathcal{G}_{2}$, any chain-recurrence class $E$ of $f$ containing a periodic point is a homoclinic class.

REMARK 2.13. The hypotheses of Proposition 2.12 are satisfied (for $f$ in $\mathcal{G}_{2}$ ), for instance, if $E$ has non-empty interior or if $E$ is isolated in $\mathcal{R}(f)$.

Combining this result with Proposition 2.9, we get:

Corollary 2.14. For any diffeomorphism $f$ in the residual set $\mathcal{G}_{2}$, any homoclinic classes which is hyperbolic is a basic piece.

Let us state a well known property of homoclinic classes which we will use later (see for instance $[\mathrm{CMP}]$ ).

REMARK 2.15. Let $M$ be a closed manifold. There is a residual subset of $\operatorname{Diff}^{1}(M)$ of diffeomorphisms $f$ such that for every periodic point $p$ of $f$ the homoclinic class $H(p, f)$ varies continuously under small $C^{1}$-perturbations.

$[\mathrm{BC}]$ shows the existence of a residual set $\mathcal{G}$ of $\operatorname{Diff}^{1}(M)$ such that, if $f \in \mathcal{G}$ and if $p$ is a (hyperbolic) periodic point whose chain-recurrence class 
$C(p)$ is isolated in $\mathcal{R}(f)$, then this class is robustly isolated: there are neighborhoods $U$ of $C(p)$ in $M$ and $\mathcal{U}$ of $f$ in $\operatorname{Diff}^{1}(M)$ such that, for any $g \in \mathcal{U}$, $\mathcal{R}(g) \cap U$ is the chain-recurrence class of the hyperbolic continuation $p_{g}$ of $p$ (in fact, the homoclinic class of $p_{g}$ ). The following proposition gives a converse of this property: for generic diffemorphisms, the non-isolated homoclinic classes are locally generically non-isolated.

Proposition 2.16. Let $M$ be a compact manifold. There is a residual subset $\mathcal{G}_{4}$ of $\operatorname{Diff}^{1}(M)$ such that any $f \in \mathcal{G}_{4}$ has the following property:

Assume that $p$ is a hyperbolic periodic point of $f$ such that the chainrecurrence class of $p$ is not isolated. Then there is a neighborhood $\mathcal{U}$ of $f$ on which the hyperbolic continuation of $p$ is well defined and such that, for any $g \in \mathcal{G}_{4} \cap \mathcal{U}$, the chain-recurrence class of $p_{g}$ is not isolated.

Proof. It is enough to prove the property for periodic orbits of period less than $n$, for any integer $n$ (that is, we will build a residual subset $\mathcal{R}_{n}$ and define the residual set $\mathcal{G}_{4}$ as the countable intersection of the $\mathcal{R}_{n}$ ). Now fix some $n \in \mathbb{N}$. There is a dense open subset $\mathcal{O}$ of $\operatorname{Diff}^{1}(M)$ such that, for any connected component $\mathcal{O}_{0}$ of $\mathcal{O}$, the periodic orbits of period less than $n$ are all hyperbolic and have a hyperbolic continuation onto the whole $\mathcal{O}_{0}$. As $\mathcal{O}$ has countably many connected components, it is enough to build, for any component $\mathcal{O}_{0}$, a residual set $\mathcal{R}_{n, \mathcal{O}_{0}}$ such that the announced properties hold in $\mathcal{R}_{n, \mathcal{O}_{0}} \cap \mathcal{O}_{0}$.

So, on $\mathcal{O}_{0}$, the periodic orbits of period less than $n$ are given by finitely many continuous functions $f \mapsto p(f)$. Thus it is sufficient to build, for any of these periodic orbits $p$, a residual set $\mathcal{R}_{n, \mathcal{O}_{0}, p}$ such that the property is satisfied on the periodic orbit $p$ for $f \in \mathcal{R}_{n, \mathcal{O}_{0}, p} \cap \mathcal{O}_{0}$.

Let $I$ be the set of diffeomorphisms $f \in \mathcal{O}_{0}$ for which the chain-recurrence class $C(p(f))$ is robustly isolated. By definition of being robustly isolated, $I$ is an open subset of $\mathcal{O}_{0}$. Write $\widetilde{I}=\mathcal{O} \backslash \bar{I}$. Then $I \cup \widetilde{I}$ is a dense open subset of $\mathcal{O}$ and therefore of $\operatorname{Diff}^{1}(M)$.

Denote by $\mathcal{G}$ the residual subset of $\operatorname{Diff}^{1}(M)$, built in [BC], on which the isolated chain-recurrence classes are robustly isolated. We define $\mathcal{R}_{n, \mathcal{O}_{0}, p}=$ $\mathcal{G} \cap(I \cup \widetilde{I})$. Consider $f \in \mathcal{R}_{n, \mathcal{O}_{0}, p} \cap \mathcal{O}_{0}$ and assume that the chain-recurrence class of $p(f)$ is not isolated. Then $f$ does not belong to $I$; consequently, $f$ belongs to $\widetilde{I}$. By definition, $\widetilde{I}$ is open and so it contains a neighborhood $\mathcal{U}$ of $f$. Therefore, for every $g \in \mathcal{R}_{n, \mathcal{O}_{0}, p} \cap \mathcal{U}$, the chain-recurrence class $C(p(g))$ is not robustly isolated (by definition of $\widetilde{I}$ ), so it is not isolated, by definition of $\mathcal{G}$.

Now, $\mathcal{R}_{n, \mathcal{O}_{0}}$ is the (finite) intersection over the periodic orbits $p$ of period less than $n$ of the residual sets $\mathcal{R}_{n, \mathcal{O}_{0}, p}$ and is therefore residual. Finally, $\mathcal{R}_{n}$ is the intersection, over all the connected components $\mathcal{O}_{0}$, of $\mathcal{R}_{n, \mathcal{O}_{0}}$. The announced residual set $\mathcal{G}_{4}$ is the intersection of all the $\mathcal{R}_{n}, n \in \mathbb{N}$. 


\section{DOMINATED SPLITTING/HOMOCLINIC TANGENCIES: \\ A LOCAL VERSION ON SURFACES}

The aim of this section is to obtain a local version of the following theorem of $[\mathrm{PS}]$ and to derive some consequences of such an extension.

Theorem 3.1 (Pujals-Sambarino, [PS]). Let $S$ be a compact surface. Then there is a dense subset $\mathcal{D}$ of $\operatorname{Diff}^{1}(S)$ such that any $f$ in $\mathcal{D}$ either is Axiom A or else exhibits a homoclinic tangency.

In order to obtain a similar local result around a chain-recurrence class, we build in the next section some geometrical filtrating neighborhoods. Using such neighborhoods we complete the dynamics outside them by adding sinks and sources; this allows us to apply Theorem 3.1 to the resulting global dynamics.

\subsection{Geometrically simple filtrating neighborhoods}

Definition 3.1. Let $f$ be a homeomorphism of a compact surface $S$. A trapping region $A$ of $f$ is said to be geometrically simple if $A$ is a compact surface with boundary and $A \backslash \operatorname{int}(f(A))$ is the finite union of disjoint annuli.

A filtrating set $U$ for $f$ is said to be geometrically simple if it is a compact surface with boundary and if there are two geometrically simple trapping regions $A$ for $f$ and $B$ for $f^{-1}$ such that:

(1) $U=A \cap B$

(2) $\mathcal{E}=A \backslash \operatorname{int}(f(A))$ and $\mathcal{S}=B \backslash \operatorname{int}\left(f^{-1}(B)\right)$ are disjoint.
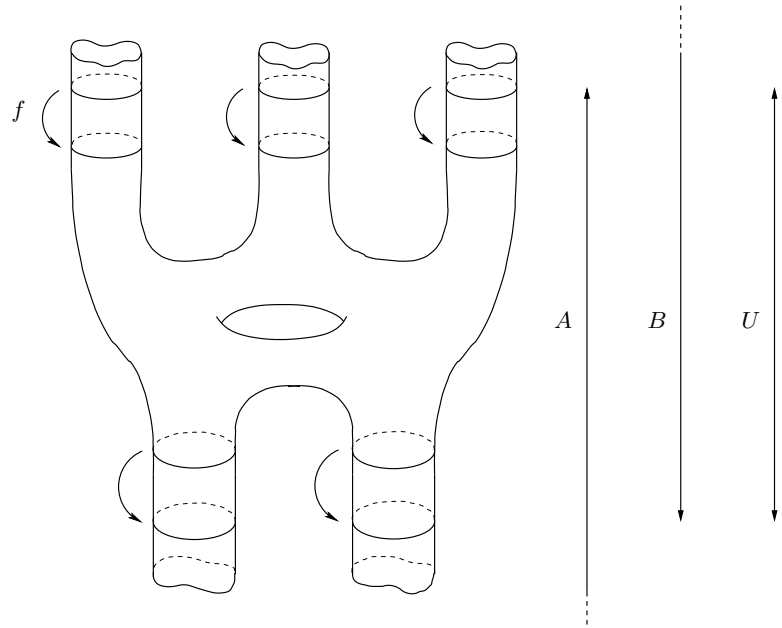

THEOREM 4. Given any homeomorphism $f$ of a compact surface $S$, any compact $f$-invariant set $\Lambda$, and any compact filtrating neighborhood $V$ 
of $\Lambda$, there is a geometrically simple filtrating set $U \subset S$ whose maximal $f$-invariant set coincides with $\Lambda$.

The proof uses the following lemma which is essentially proven in [BL, Lemme 1.3.2].

LEMma 3.2. Let $f$ be a homeomorphism of a compact surface $S$ and $W$ a trapping region for $f$. Then there is a geometrically simple trapping region $U$ such that the attractors and repellors (for $f$ ) of $U$ and $W$ coincide.

Proof. Let $\Lambda^{+}$and $\Lambda^{-}$be the attractor and repellor associated to $W$. We define the open surface $\widetilde{S}=S \backslash\left(\Lambda^{+} \cup \Lambda^{-}\right)$. By Remark 2.2(5), there exists a trapping region $\widetilde{W}$ which is a compact surface with boundary whose attractor and repellor are also $\Lambda^{+}$and $\Lambda^{-}$. Notice that any orbit of $f$ in $\widetilde{S}$ contains a unique point in $\widetilde{W} \backslash f(\widetilde{W})$ and that $\widetilde{W} \backslash \operatorname{int}(f(\widetilde{W}))$ is a compact surface with boundary whose boundary consists of the disjoint sets $\partial \widetilde{W}$ and $f(\partial \widetilde{W})$.

The orbit space of $\widetilde{S}$ is canonically identified with the closed surface $O$ obtained from $\widetilde{W} \backslash \operatorname{int}(f(\widetilde{W}))$ by gluing $\partial \widetilde{W}$ to $f(\partial \widetilde{W})$ by $f$. Note that $O$ has finitely many connected components. The canonical projection $\pi: \widetilde{S} \rightarrow O$, which associates to each point its orbit, is a covering and $f$ is an automorphism of the covering acting transitively on the fibers: for each connected component $O_{0}$ of $O, f$ generates the group of covering automorphisms (deck transformations) for $\pi^{-1}\left(O_{0}\right) \rightarrow O_{0}$.

Let $S_{0}$ be a connected component of $\widetilde{S}$ and $O_{0}=\pi\left(S_{0}\right)$ be the associated connected component of $O$. We claim that there exists $k$ such that $f^{k}\left(S_{0}\right)=S_{0}$ : Otherwise $S_{0}$ would be disjoint from $f^{i}\left(S_{0}\right)$ for each $i$ and homeomorphic to the compact surface $O_{0}$. This would imply that $S$ contains infinitely many compact surfaces, hence connected components, which is a contradiction. As a consequence, $\widetilde{S}$ has finitely many connected components. The projection $S_{0} \rightarrow O_{0}$ is an infinite cyclic covering whose automorphism group is generated by an iterate $f^{l}$ of $f$, where $l$ is the period of $S_{0}$ under the action of $f$. As the genus of $S_{0}$ is bounded (by the genus of $S$ ), $S_{0}$ is homeomorphic to the open annulus $\mathbb{S}^{1} \times \mathbb{R}$ and $O_{0}$ to the torus or to the Klein bottle. Moreover, $f^{l}$ (the automorphism of the covering) is conjugate to the translation $(x, y) \mapsto(x, y+1)$ or else to the translation composed with the symmetry: $(x, y) \mapsto(-x, y+1)$.

Summing up this argument, one sees that $\widetilde{S}$ is a finite union of disjoint cylinders $S_{i}$ homeomorphic to $\mathbb{S}^{1} \times \mathbb{R}^{1}$ which are permuted by $f$ and such that in these coordinates $f: S_{i} \rightarrow S_{j}$ expresses as $(x, y) \mapsto( \pm x, y+1)$. As a consequence, there is a family of essential circles $\mathbb{S}^{1} \times\left\{t_{i}\right\}$ in each $S_{i}$ such that the union $\widetilde{U}$ of the half-cylinders $\mathbb{S}^{1} \times\left[t_{i}, \infty[\right.$ is mapped into its interior by $f$. Furthermore, the difference $\widetilde{U} \backslash \operatorname{int}(f(\widetilde{U}))$ is a finite union of 
disjoint annuli. Notice that the intersection of $\widetilde{W}$ with any component $S_{i}$ contains a half-cylinder $\left.\mathbb{S}^{1} \times\right] a_{i}, \infty[$ and is disjoint from another half-cylinder $\left.\mathbb{S}^{1} \times\right]-\infty, b_{i}[$.

From the previous arguments, we deduce that $U=\widetilde{U} \cup \Lambda^{+}$is a compact neighborhood of $\Lambda^{+}$and hence a trapping region which is geometrically simple. Finally, the fact that the repellors of $U$ and $W$ coincide follows by construction.

Proof of Theorem 4. By Proposition 2.7, one can write the filtrating neighborhood $V$ as the intersection $A \cap B$, where $A$ and $B$ are trapping regions for $f$ and $f^{-1}$, respectively, such that the repellor associated to $A$ is disjoint from the attractor of $B$. By Lemma 3.2, there exist two geometrically simple trapping regions $\widetilde{A}$ and $\widetilde{B}$ for $f$ and $f^{-1}$, respectively, such that $A$ and $\widetilde{A}$ (resp., $B$ and $\widetilde{B}$ ) have the same attractor and repellor. As a consequence, for any $i, j \in \mathbb{Z}$, the maximal invariant sets in the filtrating sets $V=A \cap B$ and $f^{-i}(\widetilde{A}) \cap f^{j}(\widetilde{B})$ coincide (see Remark 2.2(1), (7)).

For $i$ large enough, the fundamental domain $f^{-i}(\widetilde{A}) \backslash \operatorname{int}\left(f^{-i+1}(\widetilde{A})\right)$ is contained in an arbitrarily small neighborhood of the repellor of $\widetilde{A}$, and hence of the repellor of $A$. Similarly, for $j$ large enough, the fundamental domain $f^{j}(\widetilde{B}) \backslash \operatorname{int}\left(f^{j-1}(\widetilde{B})\right)$ is contained in an arbitrarily small neighborhood of the attractor of $\widetilde{B}$, and hence of the attractor of $B$. Thus, if we define $A_{0}=f^{-i}(\widetilde{A})$ and $B_{0}=f^{j}(\widetilde{B})$, the sets $A_{0} \backslash f\left(A_{0}\right)$ and $B_{0} \backslash f^{-1}\left(B_{0}\right)$ are disjoint. Since, by our choice, $\widetilde{A}$ and $\widetilde{B}$ are geometrically simple, each of the sets $A_{0} \backslash \operatorname{int}\left(f\left(A_{0}\right)\right)$ and $B_{0} \backslash \operatorname{int}\left(f^{-1}\left(B_{0}\right)\right)$ is a finite union of disjoint annuli (these sets are homeomorphic to $\widetilde{A} \backslash \operatorname{int}(f(\widetilde{A}))$ and $\widetilde{B} \backslash \operatorname{int}\left(f^{-1}(\widetilde{B})\right)$ ).

Consequently, $U=A_{0} \cap B_{0}$ is a geometrically simple filtrating set having the same maximal invariant set as $V$.

Proposition 3.3. Let $S$ be a compact surface and $U$ a geometrically simple filtrating set of a diffeomorphism $f \in \operatorname{Diff}^{1}(S)$. Then there exist a compact surface $S_{0}$, a diffeomorphism $f_{0}$ of $S_{0}$, and a diffeomorphism $\varphi: U \rightarrow U_{0} \subset S_{0}$ such that:

- for any $x \in U \cap f^{-1}(U)$, we have $\varphi \circ f(x)=f_{0} \circ \varphi(x)$;

- the connected components of $S_{0} \backslash \operatorname{int}\left(U_{0}\right)$ are diffeomorphic to the disk $\mathbb{D}^{2}$;

- the intersection of the chain-recurrent set $\mathcal{R}\left(f_{0}\right)$ of $f_{0}$ with any connected component of $S_{0} \backslash \operatorname{int}\left(U_{0}\right)$ is a periodic point (attracting or repelling).

Proof. Write $U=A \cap B$, where $A$ and $B$ are trapping regions for $f$ and $f^{-1}$, and consider the $\operatorname{disjoint} \operatorname{sets} \mathcal{E}=A \backslash \operatorname{int}(f(A))$ and $\mathcal{S}=B \backslash \operatorname{int}\left(f^{-1}(B)\right)$, which are finite unions of disjoint annuli. 
Notice that $U \cup f(U)$ is equal to $U \cup f(\mathcal{S})$ and is obtained by gluing annuli along the boundary $\partial B \subset \partial U$. Consider the compact surface $S_{0}$ obtained by gluing disks on each boundary component of $U \cup f(U)$. There is a diffeomorphism $f^{\prime}$ of $S_{0}$ coinciding with $f$ on $U$ and satisfying the following properties:

(1) each disk attached to the boundary of $A$ (resp. $f(B)$ ) contains a unique periodic point, which is a source (resp. a sink);

(2) $\mathcal{R}\left(f_{0}\right)$ is the union of these periodic points and $\mathcal{R}(f) \cap f(U) \cap f^{-1}(U)$.

The proof of this fact is analogous to the argument in [BL, Lemme 1.3.4]. Finally, the proposition follows using the previous fact.

Consider the setting and the notations of Proposition 3.3. For the sake of simplicity, let us identify $U$ with $U_{0}=\varphi(U)$. The next proposition allows us to use the perturbations of $f_{0}$ for understanding the perturbations of $f$.

Proposition 3.4. Given any compact set $K \subset \operatorname{int}(U)$ there is a neighborhood $\mathcal{V} \subset \operatorname{Diff}^{1}\left(S_{0}\right)$ of the identity map of $S_{0}$ and a continuous map $\chi: \mathcal{V} \rightarrow \operatorname{Diff}^{1}(S)$ sending the identity map to the identity map, such that $g$ and $\chi(g)$ coincide on $K$ for any $g \in \mathcal{V}$.

The construction uses the next lemma.

Lemma 3.5. Let $M$ be a compact manifold, $U \subset M$ an open set and $K \subset U$ a compact set. Denote by $\operatorname{Diff}_{U}^{1}(M) \subset \operatorname{Diff}^{1}(M)$ the set of diffeomorphisms which are the identity outside $U$. Then there is a $C^{1}$-neighborhood $\mathcal{V} \subset \operatorname{Diff}^{1}(M)$ of the identity of $M$ and a continuous map $\psi: \mathcal{V} \rightarrow \operatorname{Diff}_{U}^{1}(M)$ which sends the identity map to the identity map such that for any $f \in \mathcal{V}$ the diffeomorphisms $f$ and $\psi(f)$ coincide on $K$.

Proof. We consider an arbitrary smooth Riemannian metric on $M$ and denote by exp: $T M \rightarrow M$ the associated exponential map. There is a small neighborhood $\widetilde{\mathcal{V}}$ of the identity of $M$ such that any $f \in \widetilde{\mathcal{V}}$ is the image under $\exp$ of a $C^{1}$-vector field $X_{f}$. Furthermore, the map $f \mapsto X_{f}$ is a homeomorphism from $\widetilde{\mathcal{V}}$ to a $C^{1}$-neighborhood $\widetilde{\mathcal{X}}$ of the zero vector field of $M$.

Let $\theta: M \rightarrow[0,1]$ be a smooth map which takes the value 0 on the complement of $U$ and the value 1 on $K$. The map $\mathcal{X}_{\theta}: f \mapsto \theta \cdot X_{f}$, defined on $\widetilde{\mathcal{V}}$, is continuous for the $C^{1}$-topology and sends the identity diffeomorphism to the zero vector field. Hence there exists a neighborhood $\mathcal{V} \subset \widetilde{\mathcal{V}}$ of the identity whose image under $\mathcal{X}_{\theta}$ is contained in $\widetilde{\mathcal{X}}$. We define $\psi$ on $\mathcal{V}$ as $f \mapsto \exp \circ \mathcal{X}_{\theta}(f)$.

Since $\theta$ is 1 on $K$ and 0 outside $U$, the diffeomorphism $\psi(f)$ coincides with $f$ on $K$ and with the identity outside $U$. Clearly, $\psi($ Id $)=$ Id.

Proof of Proposition 3.4. We apply Lemma 3.5 to the sets $K \subset U \subset S$, obtaining a neighborhood $\mathcal{V}$ of the identity of $S_{0}$ and a map $\psi: \mathcal{V} \rightarrow$ 
$\operatorname{Diff}_{U}^{1}\left(S_{0}\right)$. For each $g \in \mathcal{V}$ we define the diffeomorphism $\chi(g) \in \operatorname{Diff}^{1}(S)$ coinciding with $\psi(g)$ on $U$ (using the identification of $U$ and $U_{0}$ by $\varphi$ ) and with the identity map outside $U$.

\subsection{Dominated splitting/homoclinic tangencies: a local version.} Here is the announced local version of Theorem 3.1.

THEOREM 5. Let $S$ be a compact surface. Given any open set $\mathcal{U}$ in $\operatorname{Diff}^{1}(S)$ and any set $V$ which is a filtrating set for every $f \in \mathcal{U}$, there is a dense subset $\mathcal{D}=\mathcal{H} \cup \mathcal{T}$ of $\mathcal{U}$ such that:

(1) $\mathcal{H}$ is a $C^{1}$-open set such that for any $g \in \mathcal{H}$ the intersection $\mathcal{R}(g) \cap V$ is the union of finitely many (disjoint) hyperbolic homoclinic classes (which are basic sets);

(2) for any $g \in \mathcal{T}$ there is a homoclinic class contained in $V$ which exhibits a homoclinic tangency (whose orbit is contained in $V$ ).

Proof. Let $V$ and $\mathcal{U}$ be as in the assumptions of the theorem. Let $\mathcal{H}$ be the set of diffeomorphisms $f \in \mathcal{U}$ such that $V \cap \mathcal{R}(f)$ is hyperbolic. The set $\mathcal{H}$ is open (see Proposition 2.11). Let $\mathcal{T}$ be the set of diffeomorphisms exhibiting a homoclinic tangency in $V$. We have to show that $\mathcal{H} \cup \mathcal{T}$ is dense in $\mathcal{U}$.

Consider any $f$ in $\mathcal{U}$. Using Theorem 4 , one obtains a geometrically simple filtrating neighborhood $U$ whose maximal invariant set coincides with the maximal invariant set of $f$ in $V$. So, by Remark 2.2(2), for any $g$ sufficiently close to $f$ the maximal invariant sets of $g$ in $U$ and in $V$ coincide. So it is sufficient to prove the theorem for the set $U$.

Proposition 3.3 introduces a compact surface $S_{0}$, a diffeomorphism $f_{0}$ of $S_{0}$, and a conjugation $\varphi$. For the sake of simplicity, as above, we identify $U \subset S$ with its image $\varphi(U)=U_{0} \subset S_{0}$. We consider the compact set $K=f(U) \cap f^{-1}(U)$, which is contained in the interior of $U$ since $U$ is a filtrating set. We denote by $\mathcal{V}$ the neighborhood of the identity of $S_{0}$ and by $\chi: \mathcal{V} \rightarrow \operatorname{Diff}^{1}(S)$ the map given by Proposition 3.4.

Any diffeomorphism $g_{0} \in \operatorname{Diff}^{1}\left(S_{0}\right)$ close enough to $f_{0}$ can be written in the form $g_{0}=h_{0} \circ f_{0}$ for some $h_{0} \in \mathcal{V}$. Define $\Theta\left(g_{0}\right)$ by $\Theta\left(g_{0}\right)=\chi\left(h_{0}\right) \circ f$. This map is continuous and sends $f_{0}$ to $f$.

By Theorem 3.1, there exists $g_{0} \in \operatorname{Diff}^{1}\left(S_{0}\right)$ arbitrarily $C^{1}$-close to $f_{0}$ such that $g_{0}$ either is Axiom A or else exhibits a homoclinic tangency. Let us show that $g=\Theta\left(g_{0}\right)$ belongs to $\mathcal{H} \cup \mathcal{T}$.

By continuity of $\Theta$ and Remark 2.2(2), for every $g_{0}$ sufficiently close to $f_{0}, U$ is a filtrating set of $g=\Theta\left(g_{0}\right)$. Moreover, by Remark 2.2(4), the maximal invariant sets of $g$ in $U$ and $K$ coincide. As a consequence, the intersection $\mathcal{R}(g) \cap U$ is the union of the chain-recurrence classes contained 
in $K$ (see Remark 2.2(6)). Finally, by construction, the dynamics of $g$ and $g_{0}$ in $K$ coincide (i.e. they are conjugate by the identification $\varphi$ ).

We first consider the case where $g_{0}$ is Axiom A. Then $\mathcal{R}(g) \cap U$ is hyperbolic since $\mathcal{R}\left(g_{0}\right) \cap U$ is hyperbolic. Thus, by Proposition 2.11, we get $g \in \mathcal{H}$.

In the case where $g_{0}$ exhibits a homoclinic tangency at some point $q$ one sees that the whole orbit of $q$ is contained in $K$ : note first that $q$ is non-periodic and chain-recurrent, and the chain-recurrent set of $f_{0}$ (thus of $g_{0}$ ) outside $K$ is a finite union of hyperbolic sinks and sources. Since $K$ is a filtrating set of $f_{0}$, this remains true for $g_{0}$ close enough to $f_{0}$. Therefore the orbit of $q$ under $g_{0}$ is contained in $K$. Consequently, $g=\Theta\left(g_{0}\right)$ also exhibits a homoclinic tangency at $q$ and its orbit remains in $U$, hence in $V$, that is, $g \in \mathcal{T}$.

\subsection{Consequences of the local dichotomy}

COROLlary 3. For any compact surface $S$ there is a residual part $\mathcal{G}_{5}$ of $\operatorname{Diff}^{1}(S)$ such that every chain-recurrence class $E$ of any $f \in \mathcal{G}_{5}$ has the following property:

- if $E$ admits a dominated splitting then it is a hyperbolic basic piece;

- if E has no dominated splitting then it is accumulated by homoclinic tangencies: for any neighborhoods $U$ of $E$ in $S$ and $\mathcal{U}$ of $f$ in $\operatorname{Diff}^{1}(S)$ there exist a diffeomorphism $g \in \mathcal{U}$ and a homoclinic class $H(P, g)$ of $g$ which is contained in $U$ and exhibits a homoclinic tangency.

The proof will use the following lemma:

LEMMA 3.6. Let $V$ be a compact subset of a compact surface $S$. Then there exists a dense open subset $\mathcal{O}(V) \subset \operatorname{Diff}^{1}(S)$ which decomposes as $\mathcal{O}(V)=\mathcal{H}(V) \cup \mathcal{W}(V) \cup \mathcal{S}(V)$ such that:

(1) the sets $\mathcal{H}(V), \mathcal{W}(V)$ and $\mathcal{S}(V)$ are open and pairwise disjoint;

(2) for any $f$ in $\mathcal{H}(V)$, the set $V$ is a filtrating set of $f$ and the intersection of $\mathcal{R}(f)$ with $V$ is hyperbolic;

(3) for any $f \in \mathcal{W}(V)$, the set $V$ is a filtrating set of $f$ and there exists $g$ arbitrarily $C^{1}$-close to $f$ which exhibits a homoclinic tangency inside $V$;

(4) for any $f$ in $\mathcal{S}(V)$, the set $V$ is not a filtrating set of $f$.

Proof. Let $\mathcal{U}(V)$ be the set of $f \in \operatorname{Diff}^{1}(S)$ such that $V$ is a filtrating set of $f$. By Remark 2.2(2), the set $\mathcal{U}(V)$ is open in $\operatorname{Diff}^{1}(S)$. The set $\mathcal{S}(V)$ is the interior of $\operatorname{Diff}^{1}(S) \backslash \mathcal{U}(V)$.

Let $\mathcal{H}(V)$ be the set of diffeomorphisms $f \in \mathcal{U}(V)$ such that the intersection of $\mathcal{R}(f)$ with $V$ is hyperbolic. This set is open by Proposition 2.11. 
We define $\mathcal{W}(V)$ as $\mathcal{U}(V) \backslash \overline{\mathcal{H}(V)}$. By construction, the three sets $\mathcal{H}(V)$, $\mathcal{W}(V)$, and $\mathcal{S}(V)$ are open and pairwise disjoint. Moreover, their union is dense in $\operatorname{Diff}^{1}(S)$. It remains to show that any diffeomorphism in $\mathcal{W}(V)$ may be approximated by a diffeomorphism which exhibits a homoclinic tangency inside $V$.

We apply Theorem 5 to $V$ and the open set of diffeomorphisms $\mathcal{W}(V)$. Since for any diffeomorphism $f$ in $\mathcal{W}(V)$ the intersection $\mathcal{R}(f) \cap V$ is not hyperbolic, Theorem 5 ensures the existence of a dense subset of diffeomorphisms of $\mathcal{W}(V)$ which exhibit a homoclinic tangency inside $V$.

Proof of Corollary 3. We consider the countable family $\left\{V_{n}\right\}$ of compact sets of $S$ given by Proposition 2.6. Using Lemma 3.6 we get the residual set $\mathcal{G}_{5}=\bigcap_{n} \mathcal{O}\left(V_{n}\right)$. Let $E$ be a chain-recurrence class of $f \in \mathcal{G}_{5}$.

First, assume that $E$ has a dominated splitting. Since a dominated splitting always extends to a neighborhood (see Remark 1.6(2)), there exists a neighborhood $U$ of $E$ such that the maximal invariant set of $f$ in $U$ admits a dominated splitting. By Proposition 2.4, $E$ admits a filtrating neighborhood $V$ contained in $U$. By Proposition 2.6, there exists a filtrating neighborhood $V_{n}$ of $E$ contained in $U$. Since the existence of a dominated splitting is a robust property (see Remark 1.6(3)), by Remark 2.2(2), there exists a neighborhood $\mathcal{U}$ of $f$ in $\operatorname{Diff}^{1}(S)$ such that, for any $g \in \mathcal{U}$, the set $V_{n}$ is a filtrating set for $g$ and the maximal invariant set $\Lambda_{g}$ of $g$ in $V_{n}$ has a dominated splitting. Consequently, $g$ does not exhibit any homoclinic tangency in $V_{n}$. This shows that $f$ belongs neither to $\mathcal{S}\left(V_{n}\right)$ nor to $\mathcal{W}\left(V_{n}\right)$. Hence $f$ belongs to $\mathcal{H}\left(V_{n}\right)$ and $E$ is hyperbolic. Finally, by Proposition 2.9, it is a basic piece.

Let us now analyze the case where $E$ has no dominated splitting. Consider neighborhoods $U$ of $E$ and $\mathcal{U}$ of $f$. As above, there exists a neighborhood $V_{n} \subset U$ of $E$ which is a filtrating set. By construction, $f$ does not belong to $\mathcal{S}\left(V_{n}\right)$. Since $E$ has no dominated splitting, $f$ does not belong to $\mathcal{H}\left(V_{n}\right)$. Hence $f$ belongs to $\mathcal{W}\left(V_{n}\right)$ and may be approximated by a diffeomorphism $g \in \mathcal{U}$ which exhibits a homoclinic tangency in $V_{n} \subset U$.

\section{PROOF OF THEOREM 2 AND COROLLARY 1}

Proof of Theorem 2. We need the following dichotomy for chain-recurrence classes of $C^{1}$-generic diffeomorphisms which generalizes a similar dichotomy for homoclinic classes in [BDP].

THEOREM $4.1([\mathrm{ABC}])$. There is a residual part $\mathcal{G}_{3}$ of $\operatorname{Diff}^{1}(M)$ such that for any $f \in \mathcal{G}_{3}$ and any chain-recurrence class $E$ of $f$, the following dichotomy holds: either

(1) E admits a dominated splitting, or 
(2) $E$ is the limit of a sequence of sinks or sources for the Hausdorff topology (Newhouse phenomenon).

Let $\mathcal{G}_{3}$ and $\mathcal{G}_{5}$ be the residual subsets of $\operatorname{Diff}^{1}(S)$ given by Theorem 4.1 and Corollary 3. Let $\mathcal{G}_{0}$ be the intersection $\mathcal{G}_{0}=\mathcal{G}_{3} \cap \mathcal{G}_{5}$. We consider $f \in \mathcal{G}_{0}$ and a chain-recurrence class $E$ of $f$.

First, if $E$ has a dominated splitting then, by Corollary 3, it is a hyperbolic basic piece and hence it is isolated. If $E$ has no dominated splitting then the same corollary implies that it is accumulated by homoclinic tangencies and Theorem 4.1 shows that $E$ is the Hausdorff limit of a sequence of sinks or sources, hence $E$ is not isolated in $\mathcal{R}(f)$. This concludes the proof of Theorem 2.

Proof of Corollary 1. Suppose that $\mathcal{R}(f)$ has non-empty interior for some $f \in \mathcal{G}_{0}$. By Proposition 2.5, there is a chain-recurrence class $E$ of $f$ which has non-empty interior. Note that this interior cannot contain any sink or source, so that $E$ is not contained in the closure of sinks nor sources. By Theorem 2, this implies that $E$ is a hyperbolic basic set (with non-empty interior). Proposition 2.8 shows that $f$ is an Anosov diffeomorphism. By [F], the surface $S$ is the torus $\mathbb{T}^{2}$ and $f$ is conjugate to a linear Anosov map of $\mathbb{T}^{2}$, finishing the proof of Corollary 1 .

\section{HOMOCLINIC TANGENCIES INSIDE A PRESCRIBED HOMOCLINIC CLASS}

5.1. Homoclinic classes with small angles. The aim of this section is to create (by $C^{1}$-perturbations) homoclinic tangencies in any homoclinic class containing saddles whose stable and unstable bundles form small angles.

Proposition 5.1. Let $g$ be a diffeomorphism of a closed Riemannian surface $S$ and $H(p, g)$ a non-trivial homoclinic class. For any $\varepsilon \in] 0,2 \pi[$, if there exists a periodic point $q \in H(p, g)$ homoclinically related to the orbit of $p$ and such that the angle between the stable and unstable spaces at $q$ is smaller than $\varepsilon / 8$ then there exists a $C^{1}$-perturbation $\varphi$ of the identity of $S$ such that:

- the support of $\varphi$ is contained in an arbitrarily small neighborhood of q;

- the diffeomorphism $\varphi$ is $\varepsilon$-close to the identity in the $C^{1}$-norm;

- the diffeomorphism $h=\varphi \circ g$ has a homoclinic tangency associated to $p$ (thus the homoclinic class $H(p, h)$ contains a homoclinic tangency).

The main ingredient of the proof is the following lemma.

Lemma 5.2. Let $\varepsilon>0$ be small enough (smaller than $2 \pi$ ) and $A$ a hyperbolic automorphism of $\mathbb{R}^{2}$ such that 
- the eigenvalues $\lambda^{\mathrm{s}}, \lambda^{\mathrm{u}}$ of $A$ satisfy $\left|\lambda^{\mathrm{s}}\right|^{-1},\left|\lambda^{\mathrm{u}}\right|>5$;

- the angle between the stable space $E^{\mathrm{s}}$ and the unstable space $E^{\mathrm{u}}$ of $A$ is smaller than $\varepsilon / 8$.

Then for every pair of orbits $\mathcal{O}^{\mathrm{s}}$ in the stable space $E^{\mathrm{s}}$ and $\mathcal{O}^{\mathrm{u}}$ in the unstable space $E^{\mathrm{u}}$ of $A$ there exists a perturbation $\varphi$ of the identity of $\mathbb{R}^{2}$ such that

- $\varphi$ has compact support contained in an arbitrarily small neighborhood of $\overline{0}$ and coincides with the identity on a neighborhood of $\overline{0}$ and of the orbits $\mathcal{O}^{\mathrm{s}}$ and $\mathcal{O}^{\mathrm{u}}$

- $\varphi$ is $\varepsilon$-close to the identity in the $C^{1}$-norm;

- the diffeomorphism $\varphi \circ A$ of $\mathbb{R}^{2}$ exhibits a homoclinic tangency associated to the saddle fixed point $\overline{0}$.

Let us first prove the following lemma:

LEMMA 5.3. Let $A$ be a hyperbolic automorphism of $\mathbb{R}^{2}$ such that the eigenvalues $\lambda^{\mathrm{s}}, \lambda^{\mathrm{u}}$ of $A$ satisfy $\left|\lambda^{\mathrm{s}}\right|^{-1},\left|\lambda^{\mathrm{u}}\right|>5$. Given any pair of points $x$ in the stable space $E^{\mathrm{s}}$ of $A$ and $y$ in the unstable space $E^{\mathrm{u}}$ of $A$ there exists an arbitrarily small $t>0$ such that the strip $[t / 2, t] \times \mathbb{R}$ is disjoint from the orbits of $x$ and $y$ under $A$.

Proof. We consider the sequences $\left\{r_{i}\right\}$ and $\left\{s_{i}\right\}$ of the first coordinates of $A^{i}(x)$ and $A^{i}(y)$. We choose some arbitrarily small $r_{i}$ and assume, for instance, that $r_{i}>0$ (the argument is analogous in the other case).

By our assumption on the eigenvalues, the strip $] r_{i}, 5 r_{i}[\times \mathbb{R}$ does not contain any point of the orbit of $x$ and contains at most one point of the orbit of $y$. If it does not contain any point of the orbit of $y$ then the lemma follows for $t=2 r_{i}$. Otherwise, $] r_{i}, 5 r_{i}$ [ contains some $s_{j}$; then one of the ratios $s_{j} / r_{i}$ and $5 r_{i} / s_{j}$ is strictly larger than 2 . We can hence choose $t$ such that $[t, 2 t]$ is contained in $] r_{i}, s_{j}[\cup] s_{j}, 5 r_{i}[$.

Proof of Lemma 5.2. We fix a smooth function $\psi: \mathbb{R} \rightarrow[0,1]$ such that $\psi$ is equal to 0 outside $] 1 / 2,1$, its graph is tangent to the graph of the identity at the point $(3 / 4,3 / 4)$, and $|D \psi|$ is bounded by 4 . (One may easily build such a function by smoothing a piecewise affine function.)

We choose orthonormal coordinates of $\mathbb{R}^{2}$ such that in these coordinates the unstable space of $A$ is the axis $\mathbb{R} \times\{0\}$ and the stable space is the line $y=\sigma x$, with $\sigma>0$. By Lemma 5.3, there exists some arbitrarily small $t>0$ such that the strip $[t / 2, t] \times \mathbb{R}$ is disjoint from the orbits $\mathcal{O}^{\mathrm{s}}$ and $\mathcal{O}^{\mathrm{u}}$.

Consider the map $\widetilde{\Psi}_{\sigma}: \mathbb{R}^{2} \rightarrow \mathbb{R}^{2}$ defined by $(x, y) \mapsto(x, y+\sigma \psi(x))$. By construction, this is a diffeomorphism of $\mathbb{R}^{2}$ which coincides with the identity outside the strip $[1 / 2,1] \times \mathbb{R}$. Both $\left\|D \widetilde{\Psi}_{\sigma}-\operatorname{Id}\right\|$ and $\left\|D \widetilde{\Psi}_{\sigma}^{-1}-\operatorname{Id}\right\|$ are strictly uniformly bounded by $4 \sigma$ on $\mathbb{R}^{2}$. There exists a diffeomorphism $\Psi_{\sigma}$ of $\mathbb{R}^{2}$ with compact support contained in the strip $[1 / 2,1] \times \mathbb{R}$ such that $\left\|D \Psi_{\sigma}-\operatorname{Id}\right\|$ and $\left\|D \Psi_{\sigma}^{-1}-\operatorname{Id}\right\|$ are strictly uniformly bounded by $4 \sigma$ 
on $\mathbb{R}^{2}$ and such that $\Psi_{\sigma}$ and $\widetilde{\Psi}_{\sigma}$ coincide on a neighborhood of the segment $[1 / 2,1] \times\{0\}$.

The map $\varphi$ in the proposition is now defined by $(x, y) \mapsto t \Psi_{\sigma}(x / t, y / t)$. This is a diffeomorphism of $\mathbb{R}^{2}$ which coincides with the identity outside an arbitrarily small (for $t$ small) neighborhood of $\overline{0}$ contained in the strip $[t / 2, t] \times \mathbb{R}$. By construction, $\varphi$ is the identity on a neighborhood of $\overline{0}$ and of the orbits $\mathcal{O}^{\mathrm{s}}$ and $\mathcal{O}^{\mathrm{u}}$. Finally, the image $\varphi\left(E^{\mathrm{u}}\right)$ is tangent to the space $E^{\mathrm{S}}$ at the point $z=\varphi\left(\frac{3}{4} t, 0\right)=\left(\frac{3}{4} t, \sigma \frac{3}{4} t\right)$.

Since the $C^{1}$-norm is invariant under homotheties, both $\|D \varphi-\operatorname{Id}\|$ and $\left\|D \varphi^{-1}-\mathrm{Id}\right\|$ are strictly uniformly bounded by $4 \sigma$ on $\mathbb{R}^{2}$. Notice that $\sigma$ is the tangent of the angle $\alpha$ between the eigenspaces of $A$. By assumption, the angle $\alpha$ is less than $\varepsilon / 8<\pi / 4$ so that $\sigma$ is bounded by $2 \alpha$, which is less than $\varepsilon / 4$. Hence $\varphi$ is an $\varepsilon$-perturbation of the identity for the $C^{1}$-distance.

Set $I^{\mathrm{s}}=\{(x, \sigma x): x \in[t / 2, t]\}$. Since, by construction (Lemma 5.3), for any $n>0, A^{n}\left(I^{\mathrm{s}}\right)$ is disjoint from the strip $[t / 2, t] \times \mathbb{R}$, we see that $(\varphi \circ A)^{n}\left(I^{\mathrm{s}}\right)=A^{n}\left(I^{\mathrm{s}}\right)$, so that $I^{\mathrm{s}}$ is contained in the stable manifold of $\overline{0}$ for $\varphi \circ A$. Similarly, we set $I^{\mathrm{u}}=[t / 2, t] \times\{0\}$. For every $n>0$, we have $(\varphi \circ A)^{-n}\left(\varphi\left(I^{\mathrm{u}}\right)\right)=A^{-n}\left(I^{\mathrm{u}}\right)$, so that $\varphi\left(I^{\mathrm{u}}\right)$ is contained in the unstable manifold of $\overline{0}$ for $\varphi \circ A$. It follows that the stable and unstable manifolds of $\overline{0}$ for $\varphi \circ A$ have a tangency at $z$ (in a small neighborhood of $\overline{0})$.

Proof of Proposition 5.1. Since $H(p, g)$ is a non-trivial homoclinic class there exists some non-trivial hyperbolic basic set $K$ contained in $H(p, g)$ that contains $q$. Hence there is a family $\left\{\gamma_{n}: n \in \mathbb{N}\right\}$ of distinct periodic orbits contained in $K$ such that for each $n$ there is a point $q_{n} \in \gamma_{n}$ such that the sequence $\left\{q_{n}\right\}$ converges to $q$. This implies that

- the periods $\pi_{n}$ of the orbits $\gamma_{n}$ go to $\infty$; hence, for $n$ large enough, the stable eigenvalue $\lambda_{n}^{\mathrm{s}}$ and the unstable eigenvalue $\lambda_{n}^{\mathrm{u}}$ of $\gamma_{n}$ satisfy $\left|\lambda_{n}^{\mathrm{s}}\right|^{-1},\left|\lambda_{n}^{\mathrm{u}}\right|>5$

- the angle between the stable and unstable manifolds of $\gamma_{n}$ at $q_{n}$ is smaller than $\varepsilon / 8$ for $n$ large enough.

We consider one $q_{n}$ with $n$ large enough. By an arbitrarily small perturbation in an arbitrarily small neighborhood of $q_{n}$, we can assume that $g^{\pi_{n}}$ is linear in a neighborhood of $q_{n}$. After this small perturbation, $q_{n}$ and $p$ remain homoclinically related. We choose $x \in W^{\mathrm{s}}\left(\gamma_{n}\right) \pitchfork W^{\mathrm{u}}(p)$ and $y \in W^{\mathrm{u}}\left(\gamma_{n}\right) \pitchfork$ $W^{\mathrm{s}}(p)$. Notice that there is a small neighborhood $U$ of $q_{n}$ on which $g^{\pi_{n}}$ is linear such that the intersection of the orbit of $x$ with $U$ is contained in the local stable manifold of $q_{n}$ and has the form $\left\{g^{k \pi_{n}}\left(x_{0}\right): k \in \mathbb{N}\right\}$. Similarly, the intersection of the orbit of $y$ with $U$ is contained in the local unstable manifold of $q_{n}$ and has the form $\left\{g^{-k \pi_{n}}\left(y_{0}\right): k \in \mathbb{N}\right\}$.

By Lemma 5.2, there exists a perturbation $\varphi_{0}$ of the identity of $S$, with support in $U$, disjoint from the forward orbit of $x_{0}$ and the backward orbit 
of $y_{0}$ and such that $\varphi_{0} \circ g^{\pi_{n}}$ has a homoclinic tangency $z$ in $U$ associated to the point $q_{n}$. As the support of this perturbation is disjoint from the forward orbit of $x$, from the backward orbit of $y$, and from the whole orbits of $p$ and $q_{n}$, the points $p$ and $q_{n}$ remain homoclinically related for the diffeomorphism $\varphi_{0} \circ \mathrm{g}$. By a classical argument, an unfolding of a homoclinic tangency associated to $q_{n}$ (that is, a small perturbation in a neighborhood of $z$ ) creates a homoclinic tangency associated to $p$.

The $C^{1}$-norm of the perturbation $\varphi_{0}$ was strictly smaller than $\varepsilon$ so that the perturbation remains $\varepsilon$-close to the identity. Finally, by construction, the support of the perturbation is arbitrarily close to $q$.

\subsection{Homoclinic classes with no dominated splitting}

Proposition 5.4. Let $f$ be a diffeomorphism of a compact surface $S$ and $p$ a hyperbolic periodic point of $f$ whose homoclinic class $H(p, f)$ does not admit any dominated splitting. Fix a $C^{1}$-neighborhood $\mathcal{U}$ of $f$ and any $\alpha>0$. Then there exists a periodic point $q \in H(p, f)$ whose orbit under $f$ is homoclinically related to $p$ such that for any neighborhood $U$ of the orbit of $q$ there exists a perturbation $g \in \mathcal{U}$ of $f$, with support in $U$, such that

- the orbit of $q$ remains homoclinically related to $p$;

- the angle between the stable and unstable spaces of $q$ is bounded by $\alpha$.

Proof. One fixes some constant $\eta>0$ such that for any two diffeomorphisms $h, h^{\prime}$ which are $\eta$ - $C^{1}$-close to the identity the composed diffeomorphism $h \circ f \circ h^{\prime}$ is in $\mathcal{U}$.

Since $p$ is hyperbolic and $H(p, f)$ has no dominated splitting, the homoclinic class of $p$ is non-trivial. If the angles between the stable and unstable spaces of the periodic points whose orbits are homoclinically related to $p$ are not (uniformly) bounded from below then the proposition follows by taking $g=f$. Thus, from now on, we can assume that all these angles are greater than some $\delta>0$.

Consider a sequence $\left\{\gamma_{n}\right\}$ of periodic orbits in $H(p, f)$ which are homoclinically related to $p$, whose periods $\pi_{n}$ go to infinity, and which are dense in $H(p, f)$. Since dominated splittings extend to closures (see Remark 1.6(1)), there is no dominated splitting on the union of the orbits $\gamma_{n}$. Thus, for any $N>0$ there are a periodic orbit $\gamma_{n}$ with arbitrarily large period $\pi_{n}$ and a point $x \in \gamma_{n}$ such that for any unit vectors $u \in E^{\mathrm{S}}(x)$ and $v \in E^{\mathrm{u}}(x)$ we have

$$
2\left\|D f^{N}(x) \cdot u\right\|>\left\|D f^{N}(x) \cdot v\right\| .
$$

At each point $y \in \gamma_{n}, n \in \mathbb{N}$, one chooses unit vectors $u_{y} \in E^{\mathrm{s}}(y)$ and $v_{y} \in E^{\mathrm{u}}(y)$. Recall that the angle between the invariant spaces at each point $y \in \gamma_{n}, n \in \mathbb{N}$, is greater than $\delta$. Hence the basis transformation maps from an orthonormal basis to the basis $\left(u_{y}, v_{y}\right)$, as well as their inverses, are 
uniformly bounded in $y \in \gamma_{n}$ and $n \in \mathbb{N}$ by some constant $K>0$. If one writes $D f(y)$ in the basis $\left(u_{y}, v_{y}\right)$ of $T_{y} M$ and $\left(u_{f(y)}, v_{f(y)}\right)$ of $T_{f(y)} M$ one obtains a diagonal matrix

$$
\left(\begin{array}{cc}
a_{y} & 0 \\
0 & b_{y}
\end{array}\right) .
$$

In order to work in the basis $\left(u_{y}, v_{y}\right)$ one needs to adapt the constants $\eta$ and $\alpha$ as follows: For $\varepsilon>0$ small enough, any pair of linear maps $P, H \in$ $\mathrm{GL}(2, \mathbb{R})$ with $\|P\|<K,\left\|P^{-1}\right\|<K$, and $\|H-\mathrm{Id}\|<\varepsilon$ satisfies the inequality $\left\|P \circ H \circ P^{-1}-\mathrm{Id}\right\|<\eta$. For $\beta>0$ small enough and $P \in \operatorname{GL}(2, \mathbb{R})$ with $\|P\|<K,\left\|P^{-1}\right\|<K$, if $u, v$ are non-zero vectors whose angle is less than $\beta$, then the angle between $P(u)$ and $P(v)$ is less than $\alpha$.

We will use the following lemma, which explains how to perturb linear maps (and indeed diagonal linear maps) of $\mathbb{R}^{2}$ by diffeomorphisms in order to create small angles between the invariant manifolds while keeping control of the invariant manifolds outside a small neighborhood of $\overline{0}$. The proof of this lemma will be postponed until after the end of the proof of the proposition:

Lemma 5.5. For any $\varepsilon>0$ and any $\beta>0$ there exists $L>0$ such that for any integers $N \geq L$ and $M \geq N+2 L$ we have the following property:

Let $\left(a_{i}\right)$ and $\left(b_{i}\right)$ be two sequences of non-zero real numbers such that

- $2\left|\prod_{i=1}^{N} a_{i}\right|>\left|\prod_{i=1}^{N} b_{i}\right|$;

- $\left|\prod_{i=1}^{M} a_{i}\right|<\left|\prod_{i=1}^{M} b_{i}\right|$.

For each $i$, let $A_{i}$ be the matrix given by $\left(\begin{array}{cc}a_{i} & 0 \\ 0 & b_{i}\end{array}\right)$.

Then there exists a sequence $\left(\varphi_{i}\right)_{i \in\{1, \ldots, M\}}$ of diffeomorphisms of $\mathbb{R}^{2}$ with compact support such that, denoting by $B_{i}$ the diffeomorphism $A_{i} \circ \varphi_{i}$ for $i \in\{1, \ldots, M-1\}$ and by $B_{M}$ the diffeomorphism $\varphi_{M} \circ A_{M}$, we have the following properties:

(1) for each $i$ the diffeomorphism $\varphi_{i}$ is a perturbation of the identity which sends $\overline{0}$ to $\overline{0}$ and whose derivative is $\varepsilon$-close to the identity;

(2) the point $\overline{0}$ is a hyperbolic fixed point of $B_{M} \circ \cdots \circ B_{1}$ whose stable and unstable manifolds are the lines $\mathbb{R} \times\{0\}$ and $\{0\} \times \mathbb{R}$, respectively;

(3) the angle between the stable and unstable spaces of $\overline{0}$ for $B_{N} \circ \cdots \circ$ $B_{1} \circ B_{M} \circ \cdots \circ B_{N+1}$ is less than $\beta$.

We fix $\varepsilon>0$ and $\beta>0$ associated to $\eta$ and $\alpha$, and let $K$ be the constant which bounds the basis transformation maps, as discussed above. We choose $L$ given by Lemma $5.5, N=L$, a periodic orbit $\gamma_{n}$ with period $\pi_{n}$ larger that $N+L$, and a point $x \in \gamma_{n}$ such that $2\left\|D f^{N}(x) \cdot u_{x}\right\|>\left\|D f^{N}(x) \cdot v_{x}\right\|$. Finally, we fix $M=\pi_{n}>N+L$. 
By an arbitrarily small $C^{1}$-perturbation of $f$ in a small neighborhood of $\gamma_{n}$, one can assume that $f$ is linear in a neighborhood of each point of $\gamma_{n}$. One defines $a_{i}=a_{f^{i-1}(x)}$ and $b_{i}=b_{f^{i-1}(x)}$, for $i \in\{1, \ldots, M\}$. By construction, these numbers satisfy the hypotheses of Lemma 5.5. The lemma yields some diffeomorphisms $\varphi_{i}$ of $\mathbb{R}^{2}$. Notice that the conclusions of the lemma remain true if we conjugate all the diffeomorphisms $\varphi_{i}$ by a common homothety. Hence we can assume that the supports of the $\varphi_{i}$ 's are contained in an arbitrarily small neighborhood of $\overline{0} \in \mathbb{R}^{2}$. Consequently, using the local coordinates around the points of $\gamma_{n}$, one can consider each $\varphi_{i}$ as a diffeomorphism of $S$ coinciding with the identity outside a small neighborhood of $f^{i-1}(x)$. Denote by $g$ the diffeomorphism coinciding with $f$ outside the neighborhood of $\gamma_{n}$, with $f \circ \varphi_{i}$ in the neighborhood of $f^{i-1}(x)$ for $i \in\{1, \ldots, M-1\}$, and with $\varphi_{M} \circ f$ in the neighborhood of $f^{M-1}(x)=f^{-1}(x)$.

We now verify that $g$ has the properties in the proposition with $q=$ $f^{N}(x)$, provided that we choose the support of the $\varphi_{i}$ small enough:

- $g$ coincides with $f$ on the orbit $\gamma_{n}$;

- by the choice of $\varepsilon$, the diffeomorphism $g$ belongs to $\mathcal{U}$;

- by the choice of $\beta$, the angle between the invariant spaces of $D g^{M}$ at $f^{N}(x)$ is smaller than $\alpha$ : just note that in the basis $\left(u_{f^{N}(x)}, v_{f^{N}(x)}\right)$ this differential has the expression $B_{N} \circ \cdots \circ B_{1} \circ B_{M} \circ \cdots \circ B_{N+1}$, and the assertion follows from Lemma 5.5(3);

- recall that one can take the support of the $\varphi_{i}$ arbitrarily small; Lemma 5.5(2) asserts that the local invariant manifolds at $x$ of $g$ coincide with those of $f$ (after linearization of $f$ along $\gamma_{n}$ ), in a neighborhood of $x$ whose size does not depend on the support of the $\varphi_{i}$;

- consider two points $x_{\mathrm{s}}, x_{\mathrm{u}}$ such that $x_{\mathrm{s}}\left(\right.$ resp. $\left.x_{\mathrm{u}}\right)$ is a transverse intersection point of the local stable (resp. unstable) manifold of $x$ for $f$ with the unstable (resp. stable) manifold of the orbit of $p$ for $f$. If the support of the perturbation $\varphi_{i}$ of the identity is small enough, the positive orbits of $x_{\mathrm{u}}$ (resp. the negative orbits of $x_{\mathrm{s}}$ ) for $f$ and for $g$ coincide. As a consequence, $x$ (and then $q$ ) is homoclinically related to $p$ for $g$. This completes the proof of the proposition.

Proof of Lemma 5.5. In order to get small angles between the stable and unstable bundles we follow Mañé's arguments (see $\left[\mathrm{M}_{2}\right]$ ) which deal with matrices. However, in order to control the local invariant manifolds, we need to adapt these arguments using perturbations with compact support.

Let $\Phi$ be the matrix

$$
\Phi=\left(\begin{array}{cc}
\frac{1}{1+\varepsilon / 2} & 0 \\
0 & 1+\varepsilon / 2
\end{array}\right) .
$$

This matrix is $\varepsilon$-close to the identity. 
Denote by $D_{i}, i \in\{1, \ldots, M-1\}$, the matrix defined by $D_{i}=A_{i} \circ \Phi^{-1}$ if $i \in\{2, \ldots, L-1\}, D_{i}=A_{i} \circ \Phi$ for $i \in\{N+1, \ldots, M-2\}$, and $D_{i}=A_{i}$ in the other cases. The $D_{i}$ 's are diagonal matrices $\left(\begin{array}{cc}\alpha_{i} & 0 \\ 0 & \beta_{i}\end{array}\right)$ satisfying:

- $2\left|\prod_{i=1}^{N} \alpha_{i}\right|>(1+\varepsilon / 2)^{2(L-2)}\left|\prod_{i=1}^{N} \beta_{i}\right|$;

- $|\alpha|<|\beta|$, where $\alpha=\prod_{i=1}^{M} \alpha_{i}$ and $\beta=\prod_{i=1}^{M} \beta_{i}$.

One defines $\varphi_{1}$ as a diffeomorphism of the form $(x, y) \mapsto(x+\psi(x, y), y)$, where $\psi: \mathbb{R}^{2} \rightarrow \mathbb{R}$ has support in the unit ball, $\psi(0,0)=0,\|D \psi(x, y)\|<\varepsilon$ at each point $(x, y)$, and $(\partial / \partial y) \psi(0,0)=\varepsilon$.

Consider the image of the vertical axis $\{0\} \times \mathbb{R}$ under $D_{M} \circ \cdots \circ D_{1} \circ \varphi_{1}$. This is a graph over the second coordinate, which is mapped onto the vertical axis $\{0\} \times \mathbb{R}$ by a diffeomorphism $\widetilde{\varphi}_{M}$ of the form $(x, y) \mapsto(x+\theta(y), y)$, where $\theta(y)=-\alpha \psi(0, y / \beta)$. The function $\theta$ has support in the interval $[-|\beta|,|\beta|]$ and the modulus of its derivative $|D \theta(y)|$ is less than $|\alpha / \beta| \varepsilon \leq \varepsilon$.

This allows us to build a diffeomorphism $\varphi_{M}$, coinciding with the identity outside some compact set and with $\widetilde{\varphi}_{M}$ on $D_{M} \circ \cdots \circ D_{1} \circ \varphi_{1}(\{0\} \times \mathbb{R})$, which is $\varepsilon-C^{1}$-close to the identity.

We define $B_{1}=A_{1} \circ \varphi_{1}$ and $B_{M}=\varphi_{M} \circ A_{M}$. Notice that, by construction, $\overline{0}$ is a hyperbolic fixed point of $B_{M} \circ D_{M-1} \circ \cdots \circ D_{2} \circ B_{1}$, whose stable and unstable manifolds are the horizontal and vertical axes, respectively. As a consequence, $\overline{0}$ is a hyperbolic fixed point of $D_{N} \circ \cdots \circ D_{2} \circ B_{1} \circ B_{M} \circ$ $D_{M-1} \circ \cdots \circ D_{N+1}$ whose invariant manifolds are the images of the axes under $D_{N} \circ \cdots \circ D_{2} \circ B_{1}$. It follows that its invariant directions are generated by the vectors

$$
u=(1,0) \quad \text { and } \quad v=\left(1, v_{y}\right)=\left(1, \frac{\prod_{i=1}^{N} \beta_{i}}{\varepsilon \prod_{i=1}^{N} \alpha_{i}}\right) .
$$

Note that the modulus of $v_{y}$ is bounded by $(2 / \varepsilon)(1+\varepsilon / 2)^{-2(L-2)}$, which is arbitrarily small for large $L$; the angle between $u$ and $v$ is therefore smaller than $\beta$.

The family $B_{1}, D_{2}, \ldots, D_{M-1}, B_{M}$ has all the properties announced in the lemma, except that the $D_{i}$ are linear maps and their supports are not compact. For $i \in\{2, \ldots, M-1\}$, we choose $\varphi_{i}$ with compact support, of the form $(x, y) \mapsto\left(\lambda(x) x, \lambda^{-1}(y) y\right)$, coinciding with $\Phi$ (for $i \in$ $\{N+1, \ldots, M-1\}$ ) or $\Phi^{-1}$ (for $i \in\{2, \ldots, N\}$ ) on a very large ball $\Gamma$ centered at the origin, and whose derivative at each point is $\varepsilon$-close to the identity. We now let $B_{i}=A_{i} \circ \varphi_{i}$ for $i=2, \ldots, M-1$.

Taking the ball $\Gamma$ large enough, one verifies that $\overline{0}$ is a hyperbolic fixed point of $B_{M} \circ \cdots \circ B_{1}$ whose stable and unstable manifolds are the lines $\mathbb{R} \times\{0\}$ and $\{0\} \times \mathbb{R}$, respectively: the images $B_{1}(\{0\} \times \mathbb{R}), D_{2} \circ B_{1}(\{0\} \times \mathbb{R})$, $\ldots, D_{M-1} \circ \cdots \circ D_{2} \circ B_{1}(\{0\} \times \mathbb{R})$ of the vertical axis coincide with the 
vertical axis outside a compact domain; one simply chooses $\Gamma$ large enough to contain this compact domain.

Now the family $\varphi_{i}, i \in\{1, \ldots, M\}$, has all the announced properties.

5.3. Proof of Theorem 3. Let $f$ be a diffeomorphism of a compact surface and $p$ a hyperbolic periodic saddle whose homoclinic class $H(p, f)$ admits no dominated splitting. Fix some $\varepsilon>0$ and $\alpha=\varepsilon / 8$. By Proposition 5.4, there is an $\varepsilon$-perturbation $g_{1}$ of $f$ (coinciding with $f$ in a neighborhood of the orbit of $p$ ) such that there is a periodic saddle $q$ of $g_{1}$ homoclinically related to $p$ such that the angle between the invariant manifolds of $q$ is smaller than $\alpha$. Proposition 5.1 now yields an $\varepsilon$-perturbation $g$ of $g_{1}$ (coinciding with $g_{1}$, therefore with $f$, in a neighborhood of the orbit of $p$ ) with a homoclinic tangency associated to $p$. So we obtain an arbitrarily small $C^{1}$-perturbation $g$ of $f$ with a homoclinic tangency associated to $p$, concluding the proof of Theorem 3 .

\section{PROOF OF THE OTHER RESULTS}

Proof of Proposition 1.3. Let $f$ be a diffeomorphism of a compact surface. Assume that there is some hyperbolic set $\Lambda$ of $f$ which locally generically exhibits some tangency: there is a $C^{1}$-open neighborhood $\mathcal{U}$ of $f$ such that for every $g$ in a residual subset of $\mathcal{U}$ the stable and unstable manifolds of the continuation $\Lambda_{g}$ of $\Lambda$ are tangent at some point $x$. Notice that $x$ belongs to $W_{T}^{\mathrm{s}}\left(\Lambda_{g}\right) \cap W_{T}^{\mathrm{u}}\left(\Lambda_{g}\right)$ for some integer $T>0$. Consider, for $T \in \mathbb{N}$, the set $\mathcal{F}_{T}$ of diffeomorphisms $g \in \mathcal{U}$ having a tangency between the local invariant manifolds of size $T$ of $\Lambda_{g}$. These sets $\mathcal{F}_{T}$ are closed and their union, for $T \in \mathbb{N}$, is a residual subset in $\mathcal{U}$. One deduces that the union of the interiors of the sets $\mathcal{F}_{T}$ is a dense open subset of $\mathcal{U}$ : that is, there are robust tangencies for diffeomorphisms close to $f$.

Proof of Proposition 1.4. Recall that $\mathcal{T}$ is the $C^{1}$-open set of diffeomorphisms admitting a persistent tangency associated to some hyperbolic periodic saddle and that $\mathcal{T}_{\text {rob }} \subset \mathcal{T}$ is the open subset of diffeomorphisms admitting a robust tangency associated to some hyperbolic set. We denote by $\mathcal{T}_{\infty}$ the set $\mathcal{T} \backslash \overline{\mathcal{T}}_{\text {rob }}$.

Let $\mathcal{O}=\left\{O_{n}\right\}$ be a countable basis of open sets of $S$ and $\mathcal{F}$ the (countable) family of the closures of finite unions of sets in $\mathcal{O}$. For each $V \in \mathcal{F}$, one defines $\mathcal{U}_{V}$ as the set of diffeomorphisms $f$ for which the maximal invariant set $\Lambda(V, f)$ of $f$ in $V$ is hyperbolic. This set is open: let $f$ be in $\mathcal{U}_{V}$ and $g$ a diffeomorphism $C^{1}$-close to $f$. Then $\Lambda(V, g)$ is contained in a small neighborhood of $\Lambda(V, f)$ and hence it is hyperbolic. Let $\mathcal{V}_{V}$ be the 
$C^{1}$-interior of the complement of $\mathcal{U}_{V}$. The open set $\mathcal{U}_{V} \cup \mathcal{V}_{V}$ is dense in $\operatorname{Diff}^{1}(S)$.

For each $V \in \mathcal{F}$ and each integer $L>0$, we denote by $\mathcal{U}_{V, L}^{\text {trans }}$ the set of diffeomorphisms $f \in \mathcal{U}_{V}$ such that the local stable and unstable manifolds of $\Lambda(V, f)$ of size $L$ are transverse. We will use the following lemma whose proof we postpone.

Lemma 6.1. Each $\mathcal{U}_{V, L}^{\text {trans }}$ is open and dense in $\mathcal{U}_{V} \backslash \overline{\mathcal{T}}_{\text {rob }}$.

By this lemma, each set $\mathcal{U}_{V, L}^{\text {trans }} \cup \mathcal{V}_{V}$ is open and dense in $\operatorname{Diff}^{1}(S) \backslash \overline{\mathcal{T}}_{\text {rob }}$. Thus the intersection of this countable family (for $V \in \mathcal{F}$ and $L \in \mathbb{N}$ ) is a residual subset $\mathcal{G}$ of $\operatorname{Diff}^{1}(S) \backslash \overline{\mathcal{T}}_{\text {rob }}$. We define $\mathcal{G} \mathcal{T}_{\infty}$ as the residual subset of $\mathcal{T}_{\infty}$ obtained as the intersection of $\mathcal{G}$ with $\mathcal{T}_{\infty}$.

Let $f \in \mathcal{G} \mathcal{T}_{\infty}$ and $\Lambda$ be a hyperbolic set of $f$. There exists $V \in \mathcal{F}$ such that the maximal invariant set $\Lambda(V, f)$ contains $\Lambda$ and is hyperbolic. Thus the diffeomorphism $f$ belongs to $\mathcal{U}_{V}$. Moreover, by our choice of $\mathcal{G} \mathcal{T}_{\infty}$, for each $L$, the local invariant manifolds of $\Lambda(V, f)$ of size $L$ are transverse. Consequently, the (global) invariant manifolds of $\Lambda \subset \Lambda(V, f)$ are transverse. This completes the proof of the proposition.

Proof of Lemma 6.1. Let $f$ be a diffeomorphism, $\Lambda$ a hyperbolic set of $f$, and $\mathcal{U}$ a neighborhood of $f$ consisting of diffeomorphisms $g$ such that the hyperbolic continuation $\Lambda_{g}$ of $\Lambda$ is defined. For each integer $L>0$, we denote by $\mathcal{U}_{\Lambda, L}^{\text {trans }}$ the set of diffeomorphisms $g \in \mathcal{U}$ such that the local stable and unstable manifolds of $\Lambda_{g}$ of size $L$ are transverse. This set is open in $\mathcal{U}$ (by continuity of the local invariant manifolds). Moreover, it is also dense in $\mathcal{U} \backslash \overline{\mathcal{T}}_{\text {rob }}$ : any diffeomorphism $g$ in the interior of $\mathcal{U} \backslash \mathcal{U}_{\Lambda, L}^{\text {trans }}$ admits a robust tangency associated to $\Lambda_{g}$ (thus it belongs to $\mathcal{T}_{\text {rob }}$ ).

Let us turn to the proof of the lemma: we consider $V \in \mathcal{F}$, an integer $L>0$, and some $f \in \mathcal{U}_{V} \backslash \overline{\mathcal{T}}_{\text {rob. }}$. We choose a small neighborhood $V^{\prime} \in \mathcal{F}$ of $\Lambda(V, f)$ such that the maximal invariant set $\Lambda=\Lambda\left(V^{\prime}, f\right)$ is contained in a small neighborhood of $\Lambda(V, f)$, and hence it is hyperbolic. There is a small neighborhood $\mathcal{U}_{0}$ of $f$ such that for any $g \in \mathcal{U}_{0}$ the hyperbolic continuation $\Lambda_{g}$ of $\Lambda$ is defined.

We claim that there exists a small neighborhood $\mathcal{U} \subset \mathcal{U}_{0}$ of $f$ such that for any $g \in \mathcal{U}$ the set $\Lambda(V, g)$ is contained in $\Lambda_{g}$. By upper semicontinuity of $\Lambda(V, f)$, any orbit $O$ in $\Lambda(V, g)$ is a pseudo-orbit (with arbitrarily small jumps if $g$ is close enough to $f$ ) of $\Lambda(V, f)$. Now, by the shadowing lemma (see for example $[\mathrm{Y}]$ ), this orbit is shadowed by a (unique) orbit $O^{\prime}$ of $f$ contained in $V^{\prime}$, that is, an orbit of $\Lambda$. The orbit $O \in \Lambda(V, g)$ is shadowed by the orbit $O^{\prime} \in \Lambda$ and so, by definition and unicity of the continuation of $\Lambda$ (see $[\mathrm{Y}]$ ), it is an orbit of the continuation $\Lambda_{g}$ of $\Lambda$. We have proved that $\Lambda(V, g) \subset \Lambda_{g}$ for $g$ close to $f$. 
By the argument above, for each integer $L>0$, the set $\mathcal{U}_{\Lambda, L}^{\text {trans }}$ is dense in $\mathcal{U} \backslash \overline{\mathcal{T}}_{\text {rob }}$. For each diffeomorphism $g \in \mathcal{U}_{\Lambda . L}^{\text {trans }}$, since $\Lambda(V, g)$ is contained in $\Lambda_{g}$, the local invariant manifolds of $\Lambda(V, g)$ of size $L$ are transverse, so that $g$ also belongs to $\mathcal{U}_{V, L}^{\text {trans }}$. This proves the density of $\mathcal{U}_{V, L}^{\text {trans }}$ in $\mathcal{U}_{V} \backslash \overline{\mathcal{T}}_{\text {rob }}$.

Let us now prove the openness. We consider $f$ in $\mathcal{U}_{V, L}^{\text {trans }} \backslash \overline{\mathcal{T}}_{\text {rob }}$ and choose a small neighborhood $V^{\prime} \in \mathcal{F}$ of $V$ such that the maximal invariant set $\Lambda=\Lambda\left(V^{\prime}, f\right)$ is contained in a small neighborhood of $\Lambda(V, f)$. Hence $\Lambda$ is hyperbolic and its local invariant manifolds of size $L$ are transverse (each local manifold of $\Lambda$ is $C^{1}$-close to a local manifold of $\Lambda(V, f)$ ). Thus there exists a small neighborhood $\mathcal{U}$ of $f$ such that for each $g \in \mathcal{U}$ the hyperbolic continuation $\Lambda_{g}$ of $\Lambda$ is defined, contains the maximal invariant set $\Lambda(V, g)$ (see the arguments above), and the local invariant manifolds of size $L$ of $\Lambda_{g}$ are transverse. As a consequence, $\mathcal{U}$ is contained in $\mathcal{U}_{V, L}^{\text {trans }}$, which proves the openness and ends the proof of the lemma.

Proof of Corollary 2. Consider the residual set $\mathcal{G}_{1}$ of $\operatorname{Diff}^{1}(S)$ obtained as the intersection of the following residual sets of $\operatorname{Diff}^{1}(S): \mathcal{G}_{0}$ given by Theorem 2, $\mathcal{G}_{4}$ given by Proposition 2.16, $\mathcal{G}_{2}$ given by Proposition 2.12, and the residual set of Kupka-Smale diffeomorphisms.

Consider $f \in \mathcal{G}_{1}$ and a (hyperbolic) periodic point $p$ of $f$ whose homoclinic class $H(p, f)$ is non-isolated in $\mathcal{R}(f)$. This homoclinic class coincides with the chain-recurrence class of $p$, by Proposition 2.12. By Proposition 2.16, there exists a neighborhood $\mathcal{U}$ of $f$ such that for any $g \in \mathcal{G}_{1} \cap \mathcal{U}$ the homoclinic class of $p_{g}$ coincides with the chain-recurrence class of $p_{g}$ and is not isolated. By Theorem 2, for any $g \in \mathcal{G}_{1} \cap \mathcal{U}$ the homoclinic class of $p_{g}$ has no dominated splitting. By Theorem 3, there are diffeomorphisms $\widetilde{g}$ arbitrarily $C^{1}$-close to $g$ such that $p_{\tilde{g}}$ admits a homoclinic tangency. This yields the density in $\mathcal{U}$ of diffeomorphisms exhibiting a homoclinic tangency associated to the continuation of $p$, thus concluding the proof.

Proof of Theorem 1. Consider the open set $\mathcal{W}=\operatorname{Diff}^{1}(S) \backslash \overline{\mathcal{H} \cup \mathcal{T}}$ and let $\mathcal{G}_{0}$ and $\mathcal{G}_{1}$ be the residual subsets built in Theorem 2 and Corollary 2 . Consider $f \in \mathcal{W} \cap \mathcal{G}_{0} \cap \mathcal{G}_{1}$ and a hyperbolic periodic point $p$ of $f$. If the homoclinic class of $p$ is non-isolated then, by the definition of $\mathcal{G}_{1}, f$ exhibits a persistent homoclinic tangency associated to $p$, contradicting $f \notin \mathcal{T}$. Consequently, the homoclinic class of $p$ is isolated and is thus a hyperbolic basic piece, by the definition of $\mathcal{G}_{0}$.

We denote by $\mathcal{G} \mathcal{W}$ the set of diffeomorphisms $f \in \mathcal{W} \cap \mathcal{G}_{0} \cap \mathcal{G}_{1}$ for which each periodic point is hyperbolic and the set of periodic points is dense in the chain-recurrent set $\mathcal{R}(f)$. This set is residual in $\mathcal{W}$ by Theorem 2.2. Assume that the number of homoclinic classes of such a diffeomorphism $f \in \mathcal{G W}$ is finite. As the homoclinic classes are compact and dense 
in $\mathcal{R}(f)$, one finds that $\mathcal{R}(f)$ is the union of these homoclinic classes, hence of finitely many basic sets. This shows that $f$ satisfies Axiom $\mathrm{A}$ and the no-cycle condition, contradicting $f \notin \mathcal{H}$. So for $f$ in $\mathcal{G W}$ the number of homoclinic classes is infinite and each homoclinic class is a hyperbolic basic piece.

We conclude this paper with a discussion of the conjectures formulated in the introduction.

\section{Proposition 6.2. Conjectures 3 and 4 are equivalent.}

Proof. We first assume that Conjecture 3 is satisfied so that the open set $\mathcal{T}_{\text {rob }}$ is dense in $\mathcal{T}$. By Theorem 1, the open set $\mathcal{H} \cup \mathcal{W} \cup \mathcal{T}_{\text {rob }}$ is dense in $\operatorname{Diff}^{1}(S)$. We consider a diffeomorphism $f$ in this dense open set such that for any hyperbolic set the stable and unstable manifolds are transverse. By definition, $f$ does not belong to $\mathcal{T}_{\text {rob }}$ so that $f \in \mathcal{H} \cup \mathcal{W}$. In either case all homoclinic classes of $f$ are hyperbolic.

We now assume that Conjecture 4 is satisfied: there exists a residual subset $\mathcal{G} \subset \operatorname{Diff}^{1}(S)$ of diffeomorphisms $f$ such that, if for any hyperbolic set of $f$ its invariant manifolds are transverse, then all homoclinic classes of $f$ are hyperbolic. We can assume that this set $\mathcal{G}$ is contained in the residual set $\mathcal{G}_{0}$ given by Theorem 2 : that is, hyperbolic homoclinic classes of diffeomorphisms in $\mathcal{G}$ are (robustly) isolated.

We can also assume that the generic diffeomorphism $f$ is such that, for every (hyperbolic) periodic point $p$ of $f$, the continuation $H\left(p_{g}, g\right)$ of the homoclinic class $H(p, f)$ varies continuously under small $C^{1}$-perturbations $g$ of $f$ (see Remark 2.15).

We shall prove that $\mathcal{G} \mathcal{T}_{\infty} \cap \mathcal{G}$ is empty. Suppose, by contradiction, that there is $f \in \mathcal{G} \mathcal{T}_{\infty} \cap \mathcal{G}$. By definition of $\mathcal{G} \mathcal{T}_{\infty}$, for each hyperbolic set of $f$ its invariant manifolds are transverse, so every homoclinic class is hyperbolic, by definition of $\mathcal{G}$. This also implies that every homoclinic class $H(p, f)$ is isolated. Therefore, there are a $C^{1}$-neighborhood $\mathcal{W}$ of $f$ and a neighborhood $U$ of $H(p, f)$ such that the map $g \mapsto H\left(p_{g}, g\right)$ is continuous and $H\left(p_{g}, g\right)$ is hyperbolic and equal to the maximal invariant set of $g$ in $U$.

We claim that this implies that no diffeomorphism $g \in \mathcal{W}$ exhibits tangencies associated to $p_{g}$. Arguing by contradiction, assume that there is $g \in W$ such that $p_{g}$ has a homoclinic tangency at $x$. First, due to the hyperbolicity, the point $x$ does not belong to the homoclinic class $H\left(p_{g}, g\right)$. As this set is the maximal invariant set in $U$, this implies that we can take $x \notin U$. Unfolding this tangency, we obtain an explosion of the homoclinic class of $p_{g}$, contradicting the fact that this class is contained in $U$.

This contradicts the existence of a persistent tangency for some hyperbolic periodic point of $f$. We deduce that $\mathcal{T}_{\infty}$ is empty (since $\mathcal{G} \cap \mathcal{G} \mathcal{T}_{\infty}$ is residual in $\mathcal{T}_{\infty}$ ) so that $\mathcal{T}_{\text {rob }}$ is dense in $\mathcal{T}$, which is Conjecture 3. 


\section{References}

[ABC] F. Abdenur, Ch. Bonatti, and S. Crovisier, Global dominated splitting and the $C^{1}$ Newhouse phenomenon, Proc. Amer. Math. Soc., to appear.

[ABD] F. Abdenur, Ch. Bonatti, and L. J. Díaz, Non-wandering sets with non-empty interiors, Nonlinearity 17 (2004), 175-191.

[AS] R. Abraham and S. Smale, Nongenericity of $\Omega$-stability, in: Global Analysis (Berkeley, CA, 1968), Proc. Sympos. Pure Math. 14, Amer. Math. Soc., 1970, $5-8$.

[BC] Ch. Bonatti et S. Crovisier, Récurrence et généricité, Invent. Math. 158 (2004), 33-104.

$\left[\mathrm{BD}_{1}\right]$ Ch. Bonatti and L. Díaz, Persistent nonhyperbolic transitive diffeomorphisms, Ann. of Math. 143 (1996), 357-396.

$\left[\mathrm{BD}_{2}\right] \quad-,-$, On maximal transitive sets of generic diffeomorphisms, Publ. Math. Inst. Hautes Études Sci. 96 (2002), 171-197.

[BDP] Ch. Bonatti, L. J. Díaz, and E. R. Pujals, A $C^{1}$-generic dychotomy for diffeomorphisms: weak forms of hyperbolicity or infinitely many sinks or sources, Ann. of Math. 158 (2003), 355-418.

[BDV] Ch. Bonatti, L. J. Díaz, and M. Viana, Dynamics beyond Uniform Hyperbolicity, Encyclopaedia Math. Sci. (Math. Phys.) 102, Springer, 2004.

[BL] Ch. Bonatti et R. Langevin, Difféomorphismes de Smale des surfaces, Astérisque 250 (1998).

[BV] Ch. Bonatti and M. Viana, SRB measures for partially hyperbolic attractors: the mostly contracting case, Israel J. Math. 115 (2000), 157-193.

[CMP] C. Carballo, C. Morales, and M. J. Pacífico, Homoclinic classes for $\mathcal{C}^{1}$-generic vector fields, Ergodic Theory Dynam. Systems 23 (2003), 403-415.

[Co] C. Conley, Isolated Invariant Sets and Morse Index, CBMS Reg. Conf. Ser. Math. 38, Amer. Math. Soc., Providence, RI, 1978.

[D] L. J. Díaz, Robust nonhyperbolic dynamics and heterodimensional cycles, Ergodic Theory Dynam. Systems 15 (1995), 291-315.

[DR] L. J. Díaz and J. Rocha, Partially hyperbolic and transitive dynamics generated by heteroclinic cycles, ibid. 21 (2001), 25-76.

[F] J. Franks, Anosov diffeomorphisms, in: Global Analysis (Berkeley, CA, 1968), Proc. Sympos. Pure Math. 14, Amer. Math. Soc., 1970, 61-93.

$[\mathrm{H}] \quad$ S. Hayashi, Connecting invariant manifolds and the solution of the $C^{1}$-stability and $\Omega$-stability conjectures for flows, Ann. of Math. 145 (1997), 81-137.

$\left[\mathrm{M}_{1}\right] \quad$ R. Mañé, Contributions to the stability conjecture, Topology 17 (1978), 386-396.

$\left[\mathrm{M}_{2}\right] \quad-$, An ergodic closing lemma, Ann. of Math. 116 (1982), 503-540.

[N] S. Newhouse, Nondensity of axiom A(a) on $S^{2}$, in: Global Analysis (Berkeley, CA, 1968), Proc. Sympos. Pure Math. 14, Amer. Math. Soc., 1970, 191-202.

[PV] J. Palis and M. Viana, High dimension diffeomorphisms displaying infinitely many sinks, Ann. of Math. 140 (1994), 207-250.

[P] C. Pugh, The closing lemma, Amer. J. Math. 89 (1967), 956-1009.

[PS] E. Pujals and M. Sambarino, Homoclinic tangencies and hyperbolicity for surface diffeomorphisms, ibid. 151 (2000), 961-1023.

[R] C. Robinson, Dynamical Systems: Stability, Symbolic Dynamics, and Chaos, Stud. Adv. Math., CRC Press, Boca Raton, FL, 1999.

[Sh] M. Shub, Topologically transitive diffeomorphisms of $T^{4}$, in: Lecture Notes in Math. 206, Springer, 1971, 39-42. 
[Si] R. C. Simon, A 3-dimensional Abraham-Smale example, Proc. Amer. Math. Soc. 34 (1972), 629-630.

[Sm] S. Smale, Differentiable dynamical systems, Bull. Amer. Math. Soc. 73 (1967), 747-817.

[Y] J.-C. Yoccoz, Introduction to hyperbolic dynamics, in: Real and Complex Dynamical Systems (Hillerod, 1993), NATO Adv. Sci. Inst. Ser. C Math. Phys. Sci. 464, Kluwer, Dordrecht, 1995, 265-291.

Flavio Abdenur

IMPA

Estrada dona Castorina 110

CEP 222460-320

Rio de Janeiro, RJ, Brazil

E-mail: flavio@impa.br

Christian Bonatti

CNRS - IMB, UMR 5584

BP 47870

21078 Dijon Cedex, France

E-mail: bonatti@u-bourgogne.fr
Sylvain Crovisier

CNRS - LAGA, UMR 7539

Université Paris 13

Av. J.-B. Clément

93430 Villetaneuse, France E-mail: crovisie@math.univ-paris13.fr

Lorenzo J. Díaz

Dep. Matemática PUC-Rio

Marquês de S. Vicente 225

CEP 22453-900

Rio de Janeiro, RJ, Brazil

E-mail: lodiaz@mat.puc-rio.br

Received 10 January 2005;

in revised form 30 July 2005 